\title{
CODING PHOTOGRAPHIC MEANING: \\ HOW INTERACTIVE DIGITIZED SURROGATES AFFECT PHOTOGRAPHY EXHIBITIONS
}

by

Soha El-Sabaawi, Bachelor of Arts in Film Studies, The University of Western Ontario, 2010

\author{
A thesis \\ presented to Ryerson University \\ in partial fulfillment of the \\ requirements for the degree of \\ Master of Arts \\ in the Program of
}

Film and Photographic Preservation and Collections Management

Toronto, Ontario, Canada, 2014

(C) Soha El-Sabaawi 2014 


\section{AUTHOR'S DECLARATION FOR ELECTRONIC SUBMISSION OF A THESIS}

I hereby declare that I am the sole author of this thesis. This is a true copy of the thesis, including any required final revisions, as accepted by my examiners.

I authorize Ryerson University to lend this thesis to other institutions or individuals for the purpose of scholarly research

I further authorize Ryerson University to reproduce this thesis by photocopying or by other means, in total or in part, at the request of other institutions or individuals for the purpose of scholarly research.

I understand that my thesis may be made electronically available to the public. 


\begin{abstract}
This research paper discusses the growing trend of interactive displays in art institutions as a relevant shift in the discourse on photographic literacy, and it is addressed towards curators, archivists, museum professionals, and new media artists with a specialization in photographic studies. The paper explores the concerns of digitized materiality in interactive exhibitions, virtual museums, and image databases. Four case studies will be utilized in this discussion, including the Peel Art Gallery Museum and Archives, Google Cultural Institute, Flickr: The Commons, and The Lowcounty Digital History Initiative. The relationship between digitized materials and visitors requires an ongoing review of how a diverse demographic of museumgoers read photographs and relate to them in exhibitions. This research paper utilizes topics from new media studies to better understand the implications, benefits, and drawbacks of these different types of displays, and how they fit into the narrative of photographic theory and exhibition design.
\end{abstract}




\section{ACKNOWLEDGEMENTS}

This project could not have been completed without the patience, wisdom, and guidance of my thesis advisor Dr. Bruno Lessard, and reader Dr. Izabella Pruska-Oldenhof. I would also like to thank David Harris for his encouragement and support when this project was in its early development, and Marta Braun for her assistance in its final stages.

I owe Nick Moreau, Diane Allengame, and The Peel Art Gallery Museum and Archives much gratitude for welcoming me into their archives and taking the time to assist me with my research.

Many thanks to Christopher Sawula for the pep talks, Caroline Diezyn for the cookie deliveries, Garnet for the cuddles, and my family for putting up with my absence and descent into madness. 
To Nada Al-Yousif and Walid El-Sabaawi 


\section{TABLE OF CONTENTS}

Author's Declaration $\quad$ ii

Abstract

Acknowledgements

Dedication

iii

Table of Contents

$\mathrm{V}$

List of Figures

Introduction

1

Literature Review

6

Case Study A: The Interactive Map of Peel

13

Case Study A: Figures

Case Study B: Google Cultural Institute

Case Study C: Flickr: The Commons 


\section{LIST OF FIGURES}

\section{Page Number Figure Number Title}

16

17

17

18

18

19

19

\section{A. 1}

A. 2

A. 3

A. 4

A. 5

A. 6

A. 7

B. 1

B. 2

B. 3

B. 4

B. 5

B. 6

B. 7

B. 8

B. 9

B. 10

B. 11

C. 1

C. 2

C. 3

C. 4

C.5

D. 1

D. 2

D. 3

D. 4

D. 5
A close-up of the available time periods of Peel region maps on the touchscreen table.

A visitor toggling between a map of their neighbourhood to see changes between 1857 and today.

Enlarged photographs relevant to 1925-1949.

Albums can also be viewed together to learn more about a particular event or location.

An albumen print of the Bolton Brass Band.

A digital surrogate of the albumen print of the Bolton Brass Band.

A memory game challenging visitors to memorize the locations of photos.

A screenshot taken from the front page of Google Cultural Institute.

A screenshot of the main page of Art Project.

A screenshot of the main page of Historical Moments. A screenshot of the main page of World Wonders.

A screenshot of the search results after initiating a query for "photography."

A screenshot of the opening page for exhibition "Gordon Parks and 'Harlem Gang Leader.'"

Four screenshots of the first sections to appear when scrolling through the exhibition.

A screenshot after selecting the image Untitled (1948) by Gordon Parks.

A close-up of Untitled with a user-interface display overlaying the image.

A screenshot of the information pertaining to Untitled. The final screenshot of the exhibition.

Screenshots of the main page of Flickr: The Commons

Screenshot of search results for the subject

"daguerreotype."

Screenshots of a daguerreotype titled "Unidentified

Bride."

A screenshot of "Curate the Collection."

Reproduction and wall text of The 'Bardon Aderdare' accident (1883)

A screenshot of the main collection of exhibitions.

A screenshot of the opening image for the exhibition "The Charleston Hospital Workers Movement, 1968-1969."

A screenshot of the exhibition's introductory section.

A screenshot of the exhibition.

A screenshot of the exhibition. 


\section{Introduction}

In June 2012, the Royal Ontario Museum launched Ultimate Dinosaurs: Giants

From Gondwana, an exhibition on dinosaurs that included an augmented reality

component. Visitors downloaded a mobile app from the Apple Store to their iOS devices that allowed the camera in their device to identify geotagged markers placed around the museum. Once the device recognized a marker, a life-sized 3D model of a dinosaur appeared on the visitors' screens that resembled and behaved the way that dinosaur would have when it was alive. There were also iPads stationed in the exhibition that visitors could pick up to pan the device across dinosaurs' skeletons, prompting a realistic texture of the dinosaur's flesh to appear over the skeletons. ${ }^{1}$ The result was an educational yet entertaining show that added to the growing list of museums and art galleries incorporating technology that utilizes augmented reality. ${ }^{2}$

This exhibition details what is largely understood about how interactive technology operates for modern exhibitions in art institutions. Mobile devices, responsive 3D models, and spaces constructed with virtual reality function to entertain and immerse visitors, shifting the face-value of institutions from centres that house old, delicate artifacts to ones that engage appealing, refreshing concepts with a larger community. In reality, art institutions, particularly in Canada, have little choice but to rely on building a community in order to fund their future endeavors and preserve their current collections ${ }^{3}$,

\footnotetext{
1 "iPad App Brings Ultimate Dinosaurs To Life At The ROM," YouTube video, 3:08, posted by "Kris Abel," June 14, 2012, https://www.youtube.com/watch?v=FhLMNGy_PX8

2 "Dinosaurs roar to life with museum's augmented reality app," The Globe and Mail, last modified July 16, 2012, http://www.theglobeandmail.com/technology/tech-news/dinosaurs-roar-to-life-with-museumsaugmented-reality-app/article4420174/

3 "Luminato, AGO face cuts in Ontario budget," CBC News, last modified March 28, 2012. http://www.cbc.ca/news/arts/luminato-ago-face-cuts-in-ontario-budget-1.1230120. The Ontario government cut funding for the Art Gallery of Ontario, the Royal Ontario Museum, and the McMichael Canadian Art Collection by one percent in 2012, and two percent in 2013.
} 
and incorporating excitable technology that makes these institutions unique will likely increase their financial longevity. Beyond that, interactive technology in exhibitions also increases a participatory culture in which visitors attain agency from a new museology that works with their input using strategies such as user-generated content, membership groups, and active social media, among other practices. ${ }^{4}$ The encouragement of visitor interactivity heightens with the expansion of technology and the curation of art exhibitions both to appeal to public interest and to diversify their target demographics by particularly engaging with children. Hands-on exhibits can stimulate an "edutainment," a portmanteau of education and entertainment, which can be playful and imaginative and consequently more inviting for children.

While these exhibits are massive learning environments, they can be more problematic or alienating for older museum-goers who are lured by the high-art status of cultural institutions and the authority that comes with them. The implementation of digital activity is not a new phenomenon, but one that is already present and steadily growing in most if not all institutions, from touch screen tablets functioning as wall texts to virtual tour guides. There are other pressing issues when incorporating more interactive technology into museum and art gallery exhibitions alongside the immediate gratification of visitor appeal and child entertainment, such as the materiality of the objects that are being exhibited through digital technology. When exhibition displays are interactive via technology, that process often requires the use of digital surrogates of the objects intended to be interactive because visitors are not allowed to physically touch original objects. This practice is useful for preserving the sanctity of rare, fragile, or

\footnotetext{
${ }^{4}$ Smith Bautista, Museums in the Digital Age, 27-28

${ }^{5}$ Roussou, "Learning by doing and Learning Through Play," 247.
} 
environmentally unstable objects, but also raises concerns over the status of the object on display.

The purpose of this paper is to address the concerns of digitized materiality in an interactive photographic exhibition, both physical and virtual, and to augment the discourse relevant on photographic literacy and meaning. It aims to utilize topics from new media studies to better understand the implications, benefits, and drawbacks of different types of interactive photography exhibitions. I will also explore how image meanings are contextualized and then recontextualized through user-interface designs and methods of participation. My research contains relevant theoretical and practical knowledge, including four major examples of types of digital photographic exhibitions, including 1) a multi-touch screen tablet featuring historical photographs and maps as part of a permanent exhibition at the Peel Art Gallery, Museum, and Archive (PAMA) in Brampton, Ontario; 2) the Google Cultural Institute, a resource partnering with museums, archives, and cultural institutions to bring their collections and artifacts to the public; 3 ) an online resource by Flickr named "The Commons," that allows users to access copyright-free digitized photographs from worldwide institutions as a method to encourage users to use the photographs as they see fit without legal repercussions; and 4) The Lowcountry Digital History Initiative, a digital public history project hosted by the College of Charleston that created an online platform for their historic photographs and documents to be used for research and scholarly purposes.

\section{Methodological Considerations}

The four examples mentioned above showcase how the digitization of photographic collections in different exhibition spaces recontextualizes photography in 
their separate contexts. This is to contribute to a larger framework for understanding the theoretical concerns of digital surrogates versus their original counterparts, and the impact of digitization on photographic literacy and meaning. The examples are also different from each other in order to bring relevant yet diverse issues to the forefront. The multi-touch table in PAMA is a physical installation; Google Cultural Institute works with curators to create virtual art gallery exhibitions; Flickr promotes interactivity with their visitors by prompting them to download and transform the meaning of the photographs in their own ways; and, finally, the Digital History Initiative is an academicdriven resource acting as a digitized and online archive primarily for researchers and scholars. Between the four different examples, I aim to provide the many ways that photographic meaning can be altered through digitized and virtual spaces.

With each example, I will give a detailed description of the type of interactivity, the level of accessibility, and the user-interface (UI) design. The type of interactivity is the description of what user input is necessary in order to make the exhibition work the way the curators intended. This will largely be an explanation on how the respective technology functions in its exhibition space. The level of accessibility for each exhibition is comprised of its own material spaces (i.e. where it is located, how it can be found), and will also note which audience is meant to interact with the exhibition. This will involve noting the kind of language used and who it is designed to attract to the exhibitions, as well as the prerequisite level of education needed to understand the content. Finally, by addressing the user-interface of the exhibitions, I will be detailing the overall layouts and design of each space, as well as commenting on their efficacy on providing information on the photographic objects. These three major areas of analysis will help to explain how 
technology and digital surrogates can work in discussing both the contextualization of photography exhibitions and their overall effectiveness.

In "Analysis," the final chapter following the case studies, I will position these digitized photography exhibitions in the narrative of curating new media, including a discussion of the benefits and downfalls of moving towards a digital materiality. I will also provide an analysis of the theoretical issues surrounding materiality and immateriality through studying interfaces and the overall effects on photographic literacy. As cultural institutions approach curatorial shifts and incorporate new media to assist displaying their photographic collections, there is a pressing need to discuss how technology can be more than a trending buzzword for a museum or art gallery, and how it can affect the materiality of objects. 


\section{Literature Review}

The purpose of this literature review is to compile and summarize the major topics that frame my research. These topics are: 1) how digital surrogates affect materiality in an exhibition space, and specifically the concerns with digital materiality as a form of photographic literacy; 2) how the digitized spaces of photography exhibitions, including both technology in physical spaces and online virtual museums and art galleries, respond to concerns with materiality; 3) and how to place this type of photographic literacy and exhibition in the larger narrative of curating new media. Using these major areas of concern, I aim to place photographic literacy and meaning in the context of digitized, interactive exhibition spaces using concepts from current museology and new media studies.

\section{The Beginnings of New Media}

In 1945, Vannevar Bush, an American engineer and head of the U.S. Office of Scientific Research and Development during World War II, published an article in the Atlantic Monthly titled "As We May Think," which in some ways predicted the development of what we now refer to as new media, hypertext, hyperlinked documents, the internet, and the keyword "interactivity" by describing "voice interaction, wearable information devices, and wireless data connections that are still part of our near-future vision today." The article, however, is most famously known for a term Bush coined as the Memex, which he described as

A future device for individual use, which is a sort of mechanized private file and library ... a device in which an individual stores all his books, records, and communications, and which is mechanized so that it may be consulted with exceeding speed and flexibility. It is an enlarged intimate supplement to his

\footnotetext{
${ }^{6}$ Manovich, introduction to “As We May Think,” 35.
} 
memory. ${ }^{7}$

Bush originally envisioned the Memex as a desk with two screens onto which information would be projected via microfilm and a keyboard beside the screens to navigate. The Memex would have functioned as a proto-hypertext system that one would have utilized to access a wealth of archived information at a quick speed, and it became a hypothetical model for what is now the Internet. Bush imagined this design to manufacture a method in dealing with massive amounts of information, personalizing the experience of accessing the information, and to move science away from creating weapons to co-exist with everyday society. Although the technology would have been expensive and the project itself an ambitious one, it inspired key developments in the history of new media such as Douglas Engelbart's "hypertext."

Two decades later, Bush returned to his essay in "Memex Revisited," and cited some of the technological advancements made in the time between the two pieces, including the advancement of photography through the improvement of magnetic tapes, but he approaches the topic more pragmatically. He discusses how the technology necessary to develop the Memex at the time was being used for more "glamorous" projects, like aiding businesses and increasing public appeal through the government spending millions on space: "The libraries still operate by horse-and-buggy methods, for there is no profit in libraries. ... There is no glamour about libraries, and the public do not understand that the welfare of their children depends far more upon effective libraries than it does on the collecting of a bucket of talcum powder from the moon." 8 The issue Bush writes about twenty years after "As We May Think" is still alive today, as public

\footnotetext{
${ }^{7}$ Bush, "As We May Think," 44.

8 "Memex Revisited," 88
} 
appeal and entertainment preside over the linking of various types of media in order to create a new meaning that would immensely help archives, libraries, and cultural art institutions.

Essentially, what Bush envisioned is a device holding various documents and texts that would have needed to be photographed and programmed into a microfilm reader. The users would have access to their media, but they would still be at a distance from the media's original format, affecting how the objects would be understood outside of their material context. It would have also affected how the users interacted with the information stored, as the Memex relied on user input and navigation. As users interacted with surrogates of documents that would be projected onto microfilm readers, the materiality and textuality of the documents would significantly shift.

\section{On Digital Materiality}

Digitized surrogates are frequently treated in scholarship as secondary objects functioning to enhance an original, rather than replace it, and as methods to increase intellectual access of an institution's collection. ${ }^{9}$ However, other research suggests that surrogates can be investigated more thoroughly than digitized hand-me-downs of cultural artifacts that improve overall accessibility. Joanna Sassoon discusses how "it is no longer an accepted canon that a photograph is merely a print on paper, nor is it a simple and uncomplicated translation between reality and its mechanical representation," and suggests that in seeing photographs as more than images, we can see the digitizing process as more than transferring the physical state of a textured photograph into a pixelated version. ${ }^{10}$ Sassoon also discusses the problems with viewing an object before it

\footnotetext{
${ }^{9}$ Frost, "When the Object is Digital," 241-244.

10 "Photographic Materiality in the Age of Digital Reproduction," 188.
} 
is digitized as an "original" document because, technically, the "true" form of a

photograph is its negative, and what is printed from the negative is a copy much like what would become a digital surrogate. ${ }^{11}$ Sassoon describes a photograph as an image and object that can occupy and travel across several spaces depending on its context, as summarized here:

Three important features of the photograph are central to many debates about the complexity of photographs: the materiality of the photographic object, the concept of the original photograph and the origin of photographic meaning. It is therefore appropriate to consider a photograph as a multilayered laminated object in which meaning is derived from a symbiotic relationship between materiality, content and context. From this foundation it is possible to investigate how these aspects of the photograph are altered during the digitisation process. ${ }^{12}$

The summary above is crucial in understanding how interactive exhibitions, constructed of digitized photographs, affect the materiality of photographs and reshape the object's meaning. This follows Allan Sekula's "On the Invention of Photographic Meaning," in which he argues that "it is impossible even to conceive of an actual photograph in a "free state', unattached to a system of validation and support, that is, to a discourse." ${ }^{\prime 13}$ In understanding materiality, there needs to be a knowledge of both the physical components of a photograph and its contexts, including framing, labels, etc., and where it is physically and digitally stored. From this understanding, we can learn to re-read the meaning of the photograph in its changing state(s).

\section{On Exhibitions and Virtual Museums}

The concept of interactivity in exhibitions stems from "the goals of avant-garde art from Dada onward ... to deconstruct the barrier between the viewer and the work of

\footnotetext{
${ }^{11}$ Ibid., 191.

${ }^{12}$ Ibid., 188.

13 91-92.
} 
art." ${ }^{14}$ Narrowing the gap between the viewer and artwork is a concept that has been developing in art exhibitions for several decades, from experimental installations to contemporary practices. Peter Samis describes the implementation of technology in museums as a progression in interpretation. Visitors and even staff grew to depend on wall text, extending to object labels with basic details and an accompanying paragraph, and a "monovocal ... anonymous and authoritative "museum voice""15 built from that one-way line of communication between institutions and people. When technology began entering exhibition spaces between the 1980s and 1990s, "the monopoly of the expert" began to disappear, and "the multiplication of points of view pointed to the many meanings all happening at once." 16 The authority of wall texts especially clashes with the independence of technology, and they are described as "the pins that fix the butterflies of new media to museum walls. We need to pull out those pins if new media works are to thrive." ${ }^{\prime 17}$ By means of digital networking, which is ever increasing in exhibition spaces, visitors of museums are now able "to initiate and create, collect and interpret in their own time and space, on their own terms." 18 The role of the curator has shifted from an authority or author of the "cultural freezers"19 of museum's physically and intellectually controlled environments, and has transitioned into mediators or filters ${ }^{20}$ for their audiences. Curators, instead of approaching objects to fit into their single-narrator timeline, are now working concurrently with objects, spaces, artists, and audience. Virtual museums and galleries are different from physical exhibitions with

\footnotetext{
${ }^{14}$ Coulter-Smith and Coulter-Smith, "Mapping Outside the Frame," 49.

15 "The Exploded Museum," 5.

16 Ibid., 6.

${ }^{17}$ Ippolito, "Death by Wall Label," 108.

${ }^{18}$ Parry, Recoding the Museum, 102.

19 Ibid.

${ }^{20}$ Schleiner, "Fluidities and Oppositions among Curators," 2003.
} 
interactive technology as they act as virtual tours of a collection or exhibition on the Internet. This type of digital entity does not require visitors to physically travel to an exhibition's location. There are many benefits to this type of exhibition, including accessibility for any person without the funds or abilities to travel. There are, however, concerns about the usage of virtual reality galleries. Lianne McTavish questions the mode of interactivity celebrated in this form of exhibition, stating that "Internet users do not so much 'walk' through virtual spaces as occupy fixed positions in the center of galleries ... creating the illusion that the stable viewer moves his or her "head" to survey threedimensional spaces. ${ }^{21}$ McTavish suggests that there are more similarities between the physical and virtual museum than differences, with only the material of space (constructed, physical walls versus the more seamless construction of hyperspace). However, similar to photographs requiring context in order to understand their full meaning, Andrea Bandelli argues that instead of a focus on the technology of virtual museums as a means to re-create hyper representations of existing spaces, we should read them as "social actions in space and time." 22 The social contexts of art galleries and museums in the digital world are more crucial to our understanding of them, rather than solely viewing the technology that creates them.

\section{Filling the Gaps}

This research paper aims to contribute to the writing on interactive technology in exhibitions, both in their physical and virtual worlds. What has been missing so far is a discourse on how the materiality of photographs, or the immateriality of digital photographs, is affected by these types of exhibitions, and how these exhibitions are

21 "Visiting the Virtual Museum," 33.

22 "Virtual Spaces and Museums," 152 
written about from the perspective of new media theory. Much of what is available does not discuss how digitized photographic materials are exhibited through an interactive user-interface. Rather, scholars have focused on a larger framework of knowledge management including cataloging and virtual databases to supply information on the "inside" of a museum's structural works. The focus of my research is to discuss how interactive technology utilizing digital surrogates can recontextualize objects and materials in a photographic exhibition, and, essentially, how photography and new media can meet to provide renowned institutions with different methods of curating. 


\section{Case Study A: The Interactive Map of Peel}

The Peel Art Gallery, Museum and Archives (PAMA) is a centre in Brampton, Ontario devoted to collecting materials that narrate the history of the municipalities of Brampton, Caledon, and Mississauga, better known collectively as the Peel region in the Greater Toronto Area. In one of the centre's exhibitions, a 55" multi-touchscreen built into a table displays interactive maps and photographs of the Peel region's cultural history. Visitors can select, enlarge, rotate, and flip over 1800 thumbnails on the table, as well as read informative facts about archival photographs and documents, artifacts, and films relevant to the Peel region. The thumbnails can be grouped by categories (e.g. Aboriginal, art, landscapes, sports, etc.) or by images grouped together in albums. The table also comprises 32 responsive "touch points," which could allow up to 16 visitors at once to interact with the touchscreen.

There are seven time periods visitors can choose from on the map: 1850-1874, 1875-1899, 1900-1924, 1925-1949, 1950-1974, 1975-1999, and 2000-Today (fig. A.1).

Once the visitor chooses a time period, a map representing that time period stretches to fit the screen, which visitors can also toggle with other maps to track changes across the region (fig. A.2). Then, after a map is chosen and displayed, various areas of interest appear across the screen where visitors can enlarge photographs relevant to that area (fig. A.3), turn the photographs around to read more about them, or scroll through an album of related photographs to learn more about a particular event or location (fig. A.4). The photographs include digitized postcards, albumen prints, and glass plate negatives that have been inverted into positive images, and panoramic prints.

One of the important aspects of the Interactive Map of Peel is how the technology 
can display digital surrogates of old, brittle photographs. Visitors are able to interact with images that cannot be displayed traditionally, like the discoloured albumen print of the Bolton Brass Band from 1892 in fig. A.5, which is covered in scratches and peppered with rust-coloured spots and stains, by enlarging its digital surrogate (fig. A.6) to investigate the image on a level that would not be allowed in a traditional exhibition design due to environmental conditions that would place the print in a higher risk of material and chemical deterioration. This is one example of many photographic materials in need of urgent preservation or conservation displayed throughout the table, including panoramic prints with curled edges that are weighing down the rest of the print, and brittle, chipped glass-plate negatives that have severe binder loss between the chemical emulsion and plate.

The location of the table is across from an exhibition space designed for children, and given the table's hip-level height for an average adult, the result is an interactive exhibition created with a younger demographic as its target audience. There is also an element of playfulness in the design as visitors can play a memory-based puzzle game to memorize locations of photographs before they randomly scatter across the map (fig. A.7), thus emphasizing how technology in art galleries and museums can focus on providing an "edutainment" to increase visitors' appeal. Visitors playing this game are asked to contextualize the photographs in a proper narrative to reiterate their significance for a group of locations, and the photographs become symbols of importance for points of interest. The game is an intriguing method to keep the focus on photographs as methods of representation. Instead of swiping through photographs on a touchscreen without paying much attention, visitors can recall and identify what photographs represent on a 
larger historical scale.

The written content is accessible, with no previous art history or photography background necessary, and appears to be worded for at least a seventh grade level of reading comprehension. The result of this playful, yet informative, design provides an understanding of early photography to a younger audience. Although the understanding is limited, and children will not be given a lesson on how photographs are made chemically or technologically, it is still important to note that children will be able to visually experience what old photographs look like as this technology is emulating realistic depictions of the photographic degradation process by showing objects that display a range of material loss or damage. The multi-touch table preserves a truthfulness to how the photographic medium changes over the allocated decades by guarding the materiality of the images so the visitors can see every scratch, stain, pore, and smudge. I found this particularly important because photographic exhibitions typically place an importance on artistic merit and aesthetics, whereas the Interactive Map of Peel bridges photography in a historical context that cherishes what would be deemed as unattractive in traditional art exhibitions.

The overall user-interface design creates a seamless experience between the visitor and the photographs. The table is not an intrusive third-party taking significance away from photographs, and is not compromising the sanctity of aging materials, but is being utilized to provide a historical context for photographs that have to be tucked away in storage vaults due to their conditions. As a result, the digital surrogates exhibited on this table are effectively allowing photographs to live in different, simultaneous ways. While the table is a method of display and exhibition, it also serves as a method of 
digitally preserving the materials that are concurrently being shown to an audience, as well as being stored in environmentally stable, non-toxic packaging, and cared for by professional archivists. The technology is both an interactive method of presentation and a digital method of preservation that ultimately centers on photographic history.

\section{Case Study A: Figures}

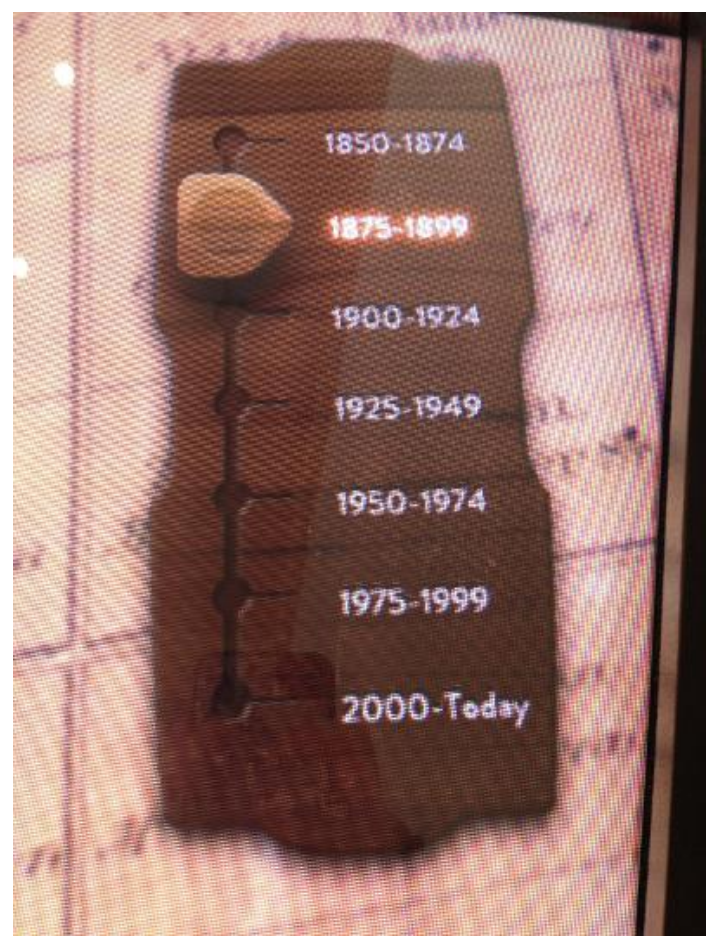

Figure A.1: A close-up of the available time periods of Peel region maps on the touchscreen table. Visitors slide the waypoint on the left that highlights a time period, and confirm which map they would like to see. Image taken by Soha El-Sabaawi. 


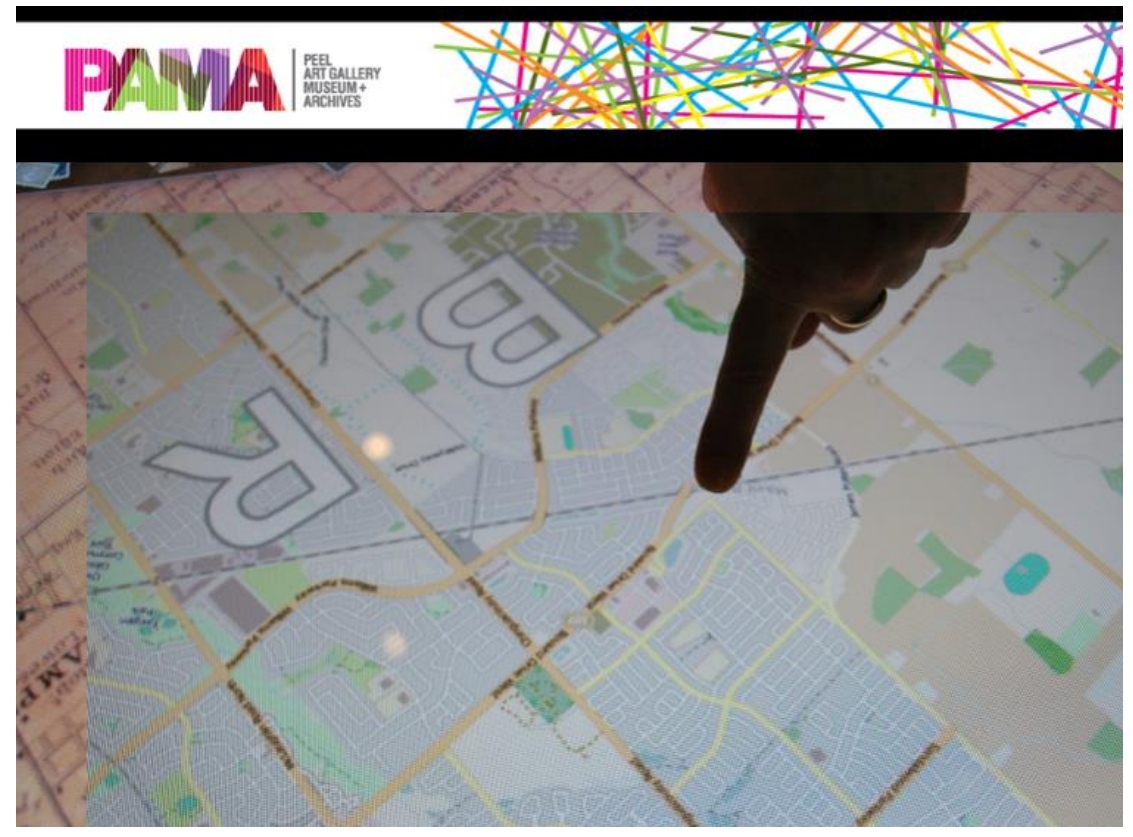

Figure A.2: A visitor toggling between a map of their neighbourhood to see changes between 1857 and today. Image courtesy of Nick Moreau and PAMA.

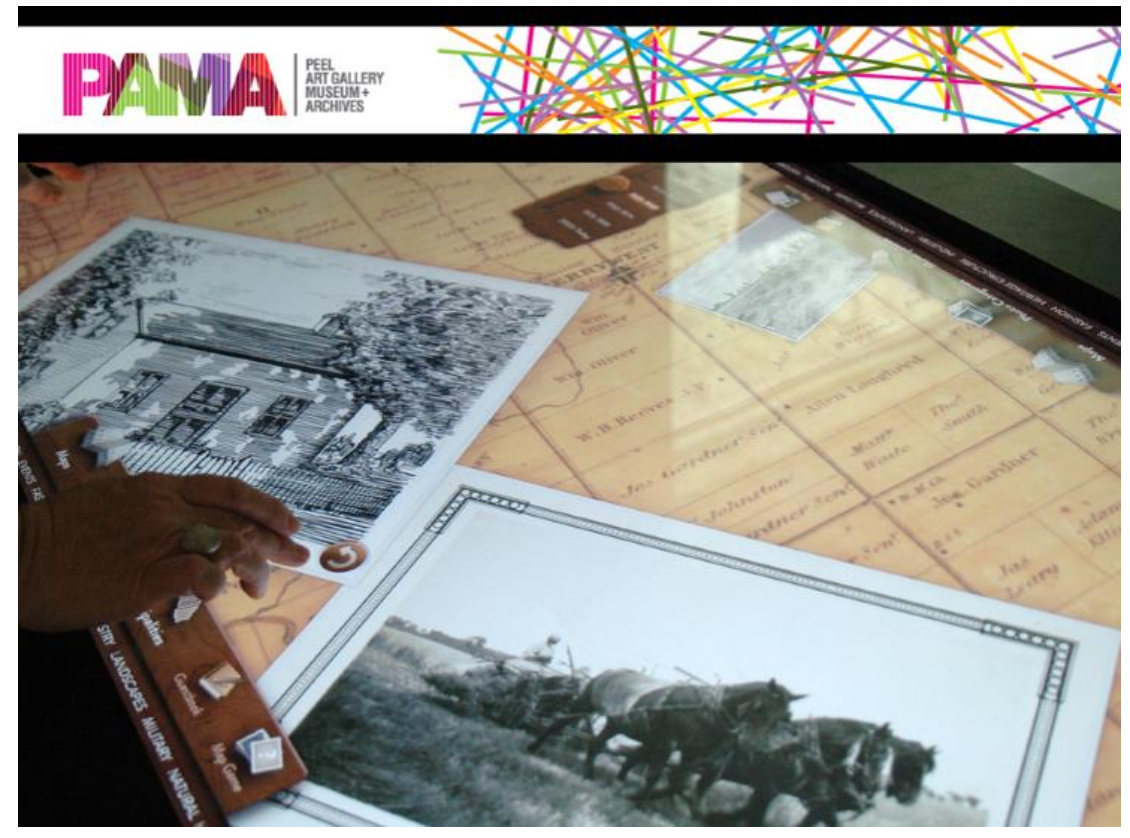

Figure A.3: Enlarged photographs relevant to 1925-1949. The white arrow with a red circular background on the bottom right of the photograph on the left prompts the photograph to turn over and display information pertinent to it. Image courtesy of Nick Moreau and PAMA. 


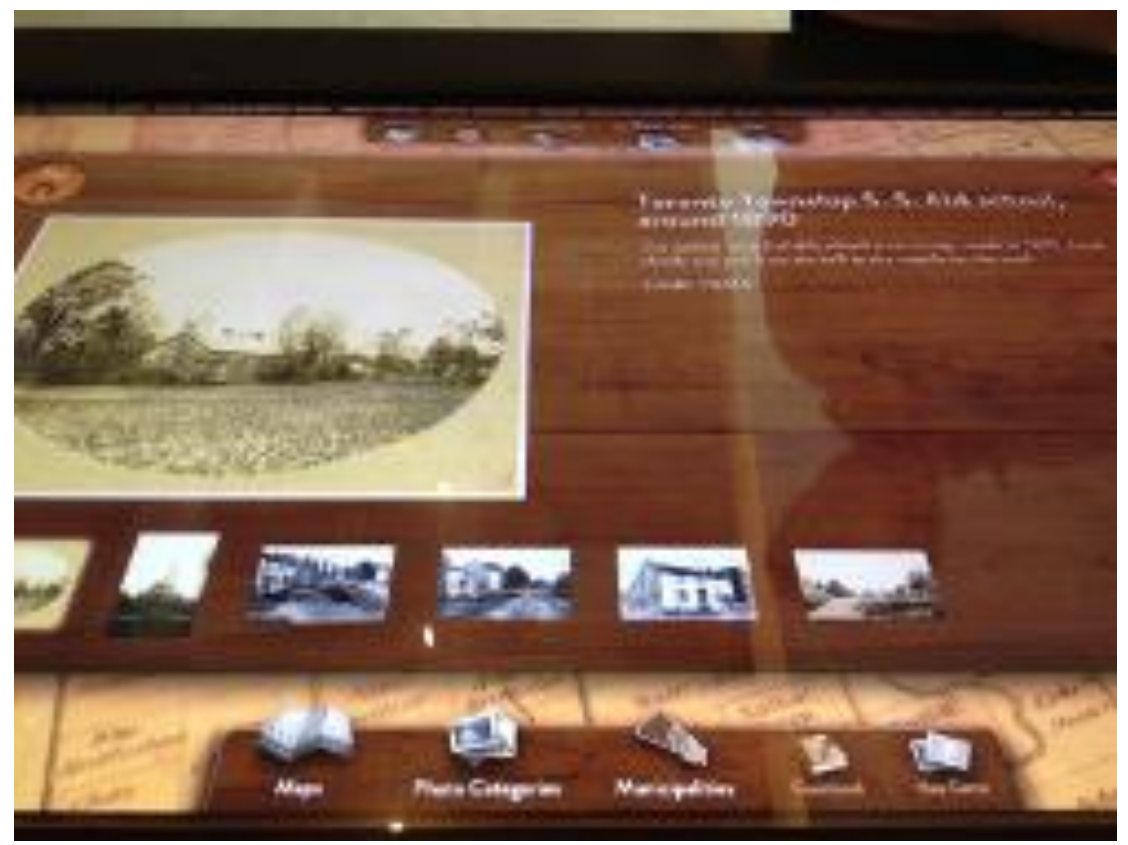

Figure A.4: Albums can also be viewed together to learn more about a particular event or location. This image particularly relays information about the Toronto Township Secondary School circa 1890. Image taken by Soha El-Sabaawi.

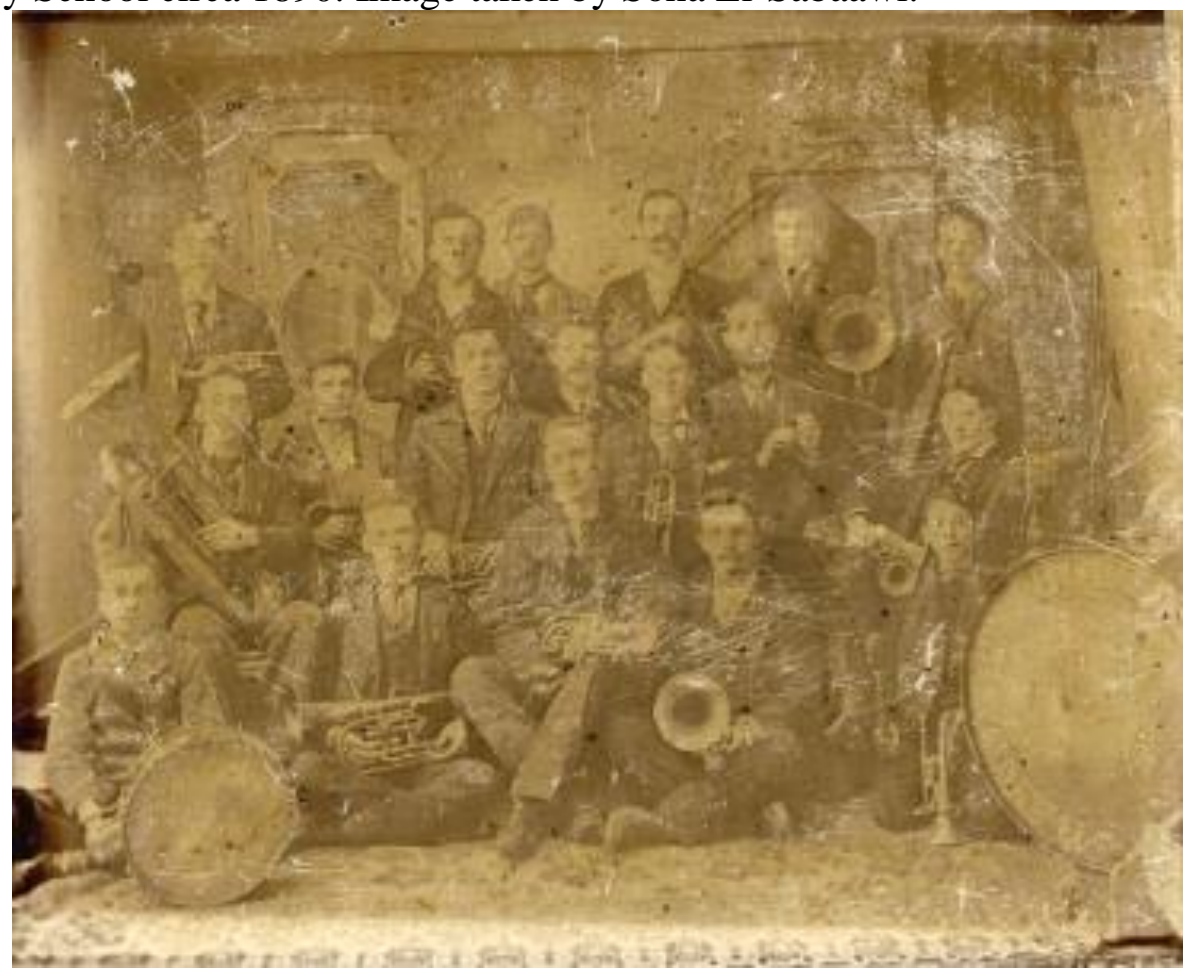

Figure A.5: An albumen print (image: $20 \times 25.2 \mathrm{~cm}$, mounted: $27.2 \times 32.8 \mathrm{~cm}$ ) of the Bolton Brass Band from 1892. Courtesy of the PAMA and the Albion-Bolton Historical Society. 


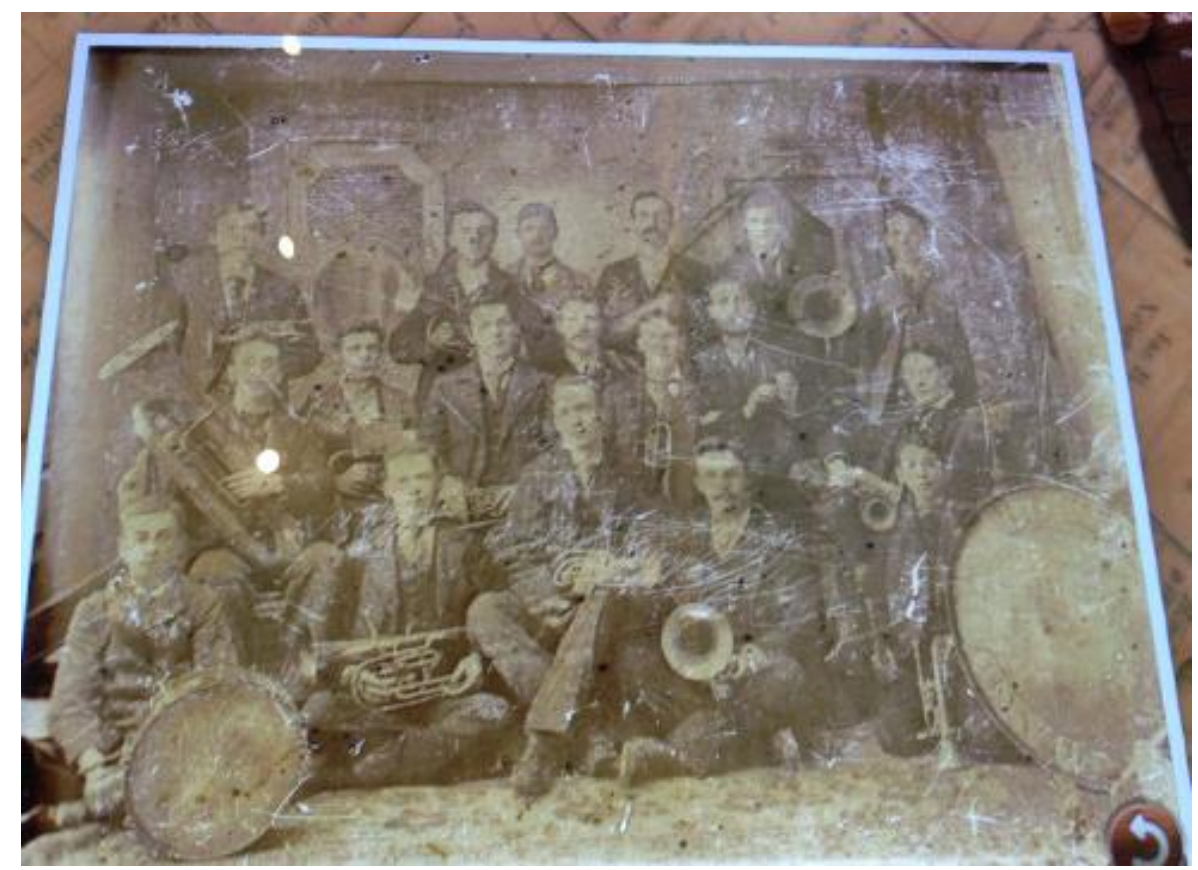

Figure A.6: A digital surrogate of the albumen print of the Bolton Brass Band (fig. A.5) taken as it appears on the Interactive Map of Peel. Image taken by Soha El-Sabaawi.

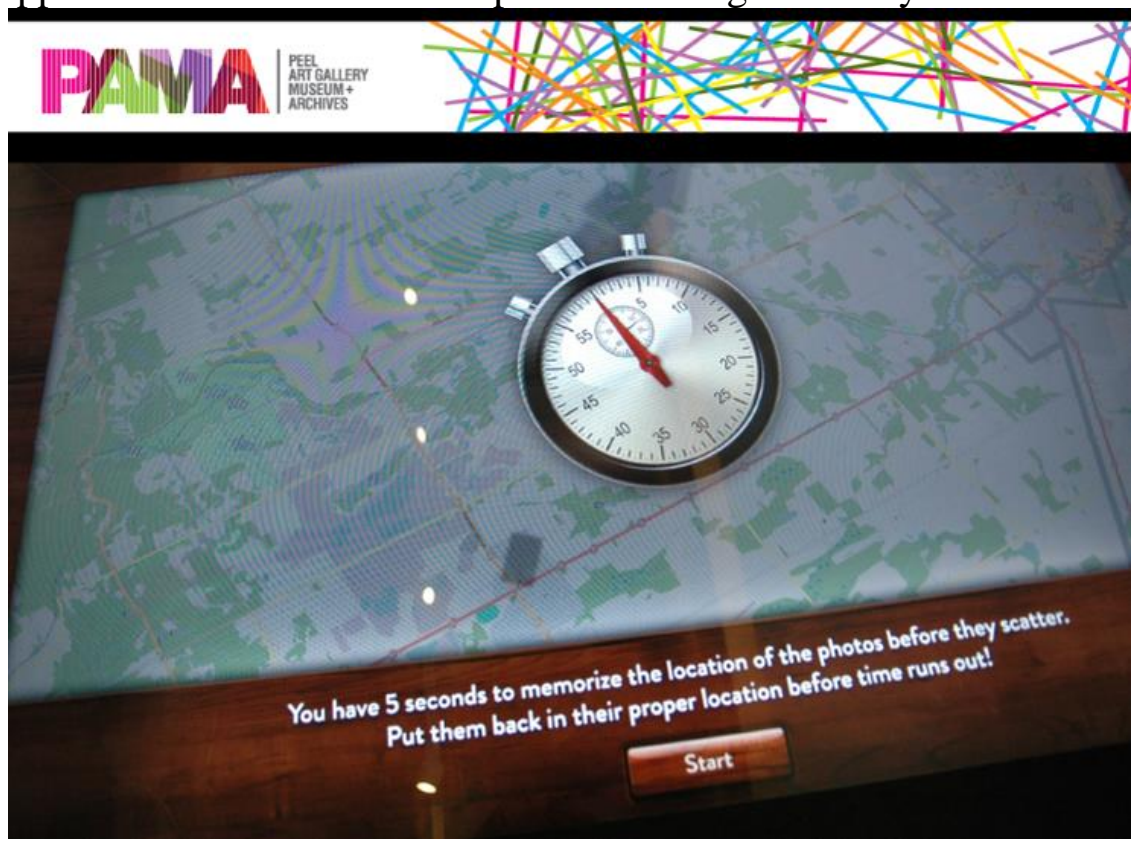

Figure A.7: A memory game challenging visitors to memorize locations of photos is a feature in the Interactive Map of Peel. Image courtesy of Nick Moreau and PAMA. 


\section{Case Study B: Google Cultural Institute}

Google Cultural Institute (GCI) is a resource featuring virtual tours, digital collections, and online exhibitions from hundreds of international cultural institutions (fig. B.1). Its mission is to "make important cultural material available and accessible to everyone and to digitally preserve it to educate and inspire future generations," and provide access to "paintings, drawings, sculptures, religious artifacts, landmarks, historic photographs and important manuscripts from collections around the world." ${ }^{23}$ The GCI initiative consists of three major sections: Art Project, Historic Moments, and World Wonders. The Art Project (fig. B.2) offers high-resolution artwork accompanied by narrated videos, audio guides, and additional notes from over 250 institutions and 6000 artists. ${ }^{24}$ Historic Moments (fig. B.3) highlights online exhibitions conceptualizing significant moments in history such as D-Day, the Civil Rights Act of 1964, the building of the Statue of Liberty, and so forth. Last, World Wonders (fig. B.4) focuses on capturing heritage sites by utilizing Google's Street View, a panoramic viewpoint controlled by user-input, and presents virtual access to historically and geographically relevant locations such as Stonehenge, Taj Mahal, and the Grand Canyon, among others.

For this case study, I will address the way GCI showcases and organizes the photographic exhibitions relevant to my research. Using the search engine, I typed in "photography" and then clicked on "exhibits," which led me to a selection of 60 exhibits related to photography (fig. B.5). To exemplify how photographic exhibitions become virtual, I chose to view "Gordon Parks and 'Harlem Gang Reader,"” (fig. B.6) based on

\footnotetext{
23 "Frequently Asked Questions," Google Cultural Institute, accessed June15, 2014, http://www.google.com/culturalinstitute/about/

24 “Art Project," Google Cultural Institute, accessed June 15, 2014
} 
the exhibition "Gordon Parks: The Making of an Argument" that was on view in the New Orleans Museum of Art from September 12, 2013 until January 19, 2014. ${ }^{25}$ Both the physical and virtual exhibitions explore Parks' first photographic essay, "Harlem Gang Leader," for Life magazine in 1948. The exhibition instructs users to either move through the exhibition by scrolling up and down on a computer mouse, or to press the left and right arrow keys on a keyboard. Users can track their progress by a bar underneath the exhibition that represents where the users are in the larger layout view. The exhibition is comprised of photographs, texts, and spreads taken from Life magazine that can each be selected for closer viewing and additional information (fig. B.7).

I chose a photograph of Leonard "Red" Jackson smoking a cigarette with his eyes closed (fig. B.8) to view the amount of information available. By scrolling with a computer mouse or using the Zoom In/Out tool overlaying the image, I was able to zoom into the image far without losing quality to further investigate its surface (fig. B.9), an impossible task in a physical exhibition without having the glass of a frame, or an institution's security personnel, obstruct the view. There is also an option to view the image without the user-interface overlaying the surface of the image (as shown in the second screenshot in fig. B.9) to create a seamless exploration of the photograph. After zooming out, I was able to obtain more information on the photograph I selected (fig. B.10) such as the description, physical format, provenance, and dimensions of the original object. There are 47 photographic objects in the exhibition, and each of them has the same feature as the photograph I just described, creating a dense amount of

\footnotetext{
25 "Gordon Parks: The Making of an Argument," New Orleans Museum of Art, last updated August 17 , 2013 http://noma.org/news/detail/222/GORDON-PARKS-THE-MAKING-OF-AN-ARGUMENT
} 
information. The final section of the exhibition is to credit and acknowledge not only those who own the copyright of the photographs, but to also recognize if other users or foundations have influenced the design of the exhibition, in this case being The Gordon Parks Foundation (fig. B.11), who contributed to some of the "wall text" in the exhibition.

There are many photographic exhibitions similar to the one dedicated to Gordon Parks such as "Nelson Mandela's Early: Life" and "The Fall of the Berlin Wall," and they are each relevant to discussing how digital materiality is being represented and understood through an engine like the Google Cultural Institute. The historical narratives displayed through GCI can be accessed anytime and anywhere as long as there is a sufficient Internet connection. That type of mobility and accessibility greatly widens the demographic able to access knowledge about influential photographers like Gordon Parks, as well as historic photography movements and topics. It is also important to note that the exhibitions through GCI are curated by cultural institutions and heavily rely on the input and contribution of the contents' legal owners. While the method of display and interface is interactive and does not take place in a museum or art gallery, the content produced still values a curatorial and institutional voice. 


\section{Case Study B: Figures}

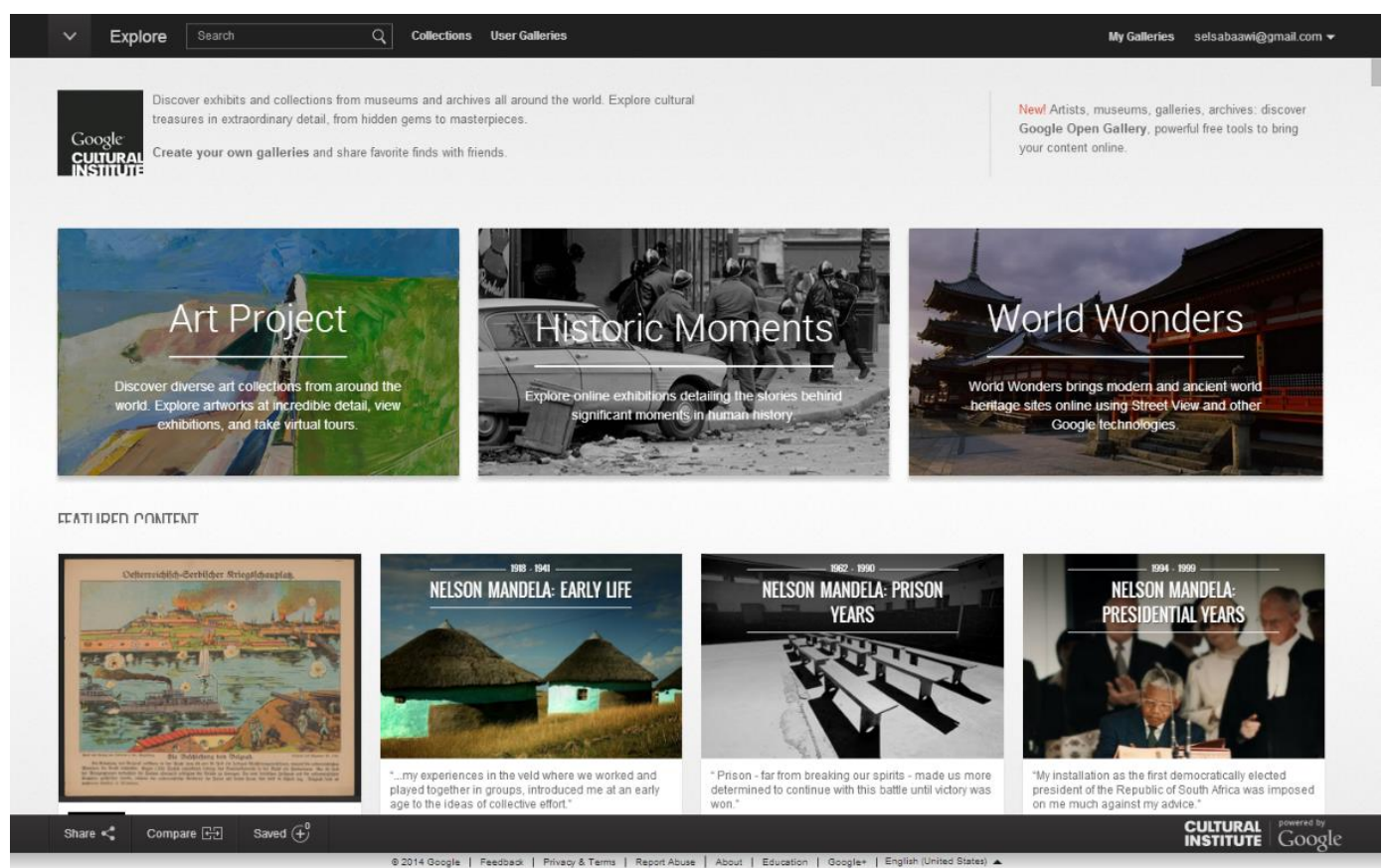

Figure B.1: A screenshot taken from the front page of Google Cultural Institute. Image taken by Soha El-Sabaawi.

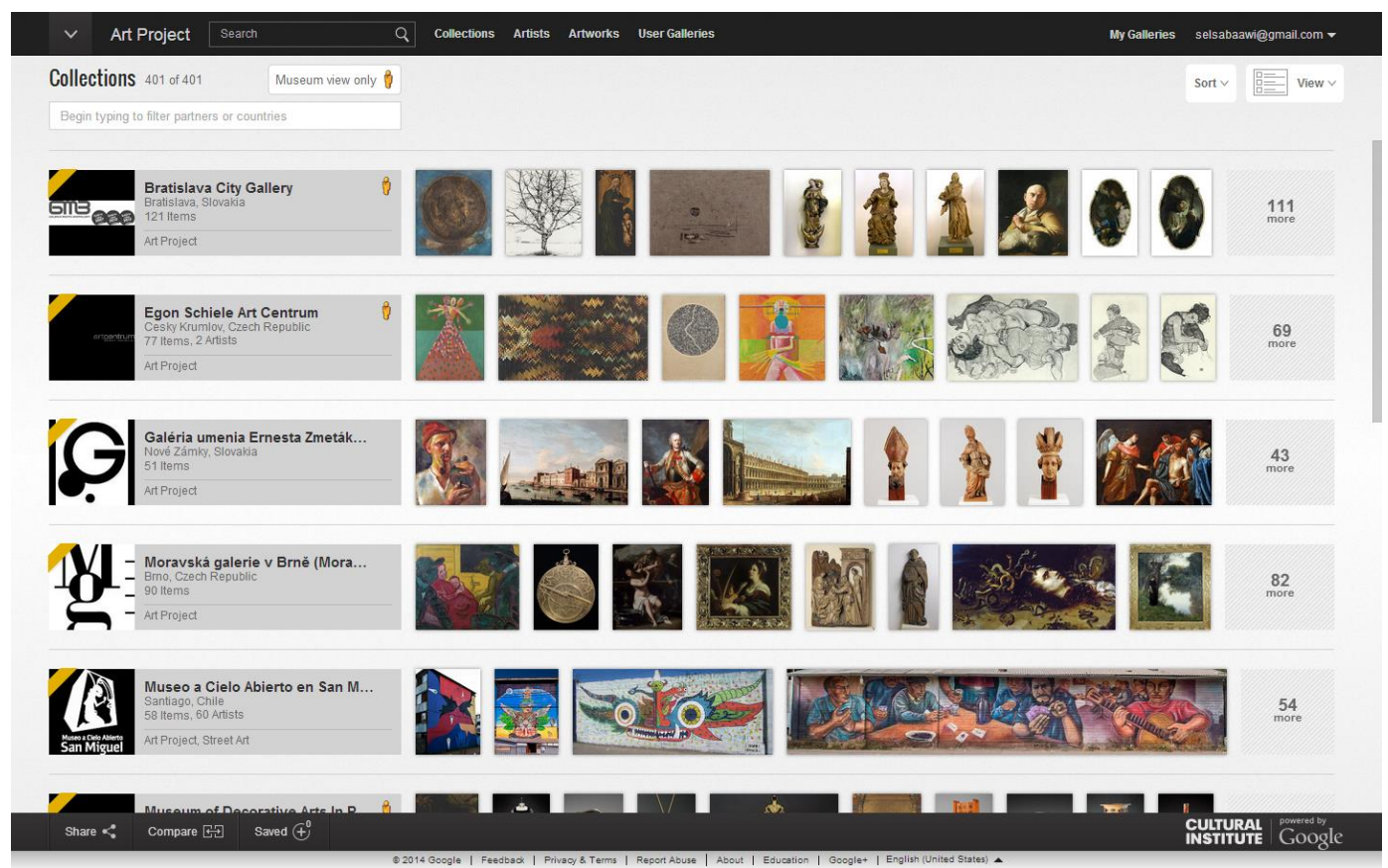

Figure B.2: A screenshot of the main page of Art Project. Image taken by Soha ElSabaawi. 


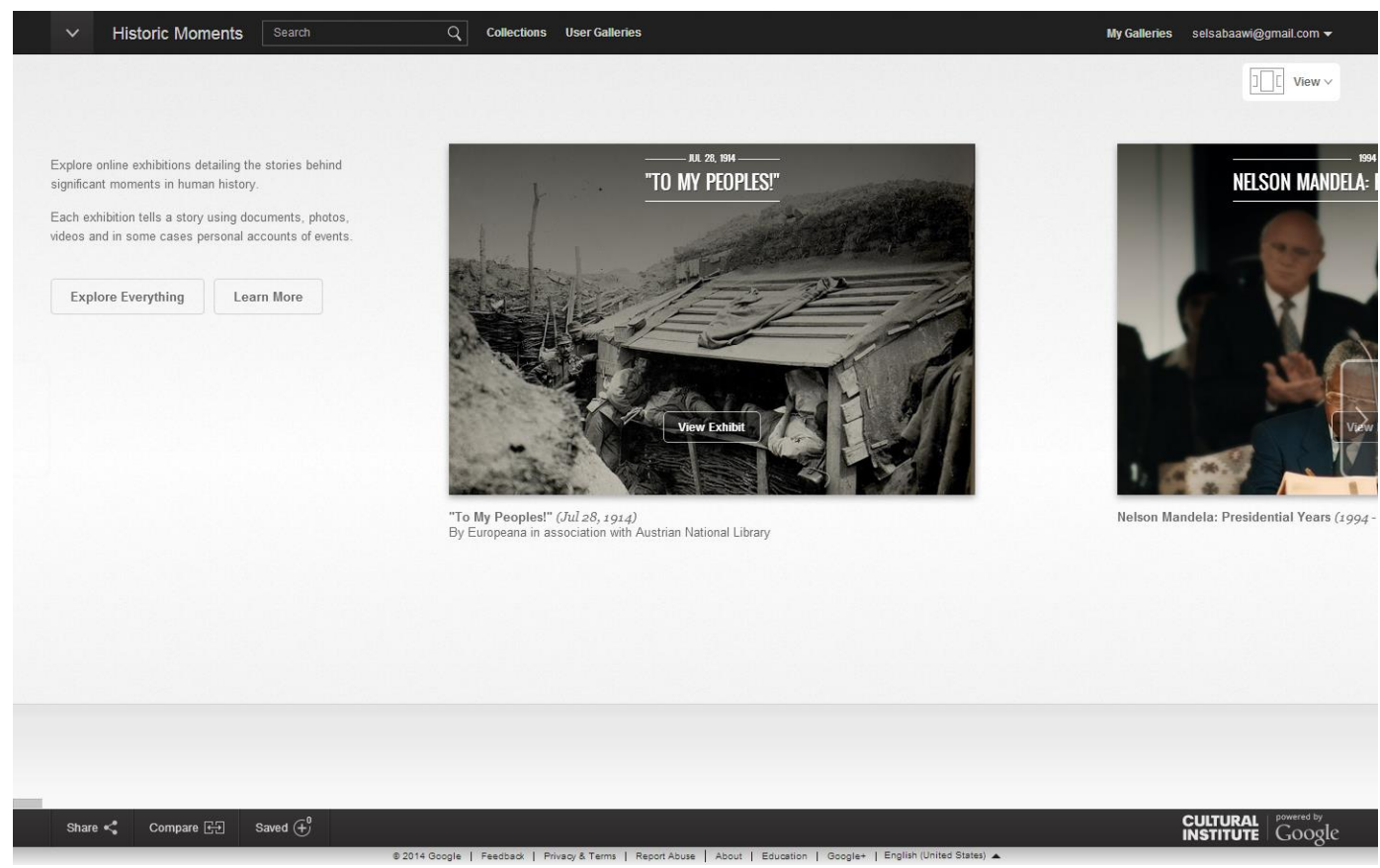

Figure B.3: A screenshot of the main page of Historical Moments. Image taken by Soha El-Sabaawi.

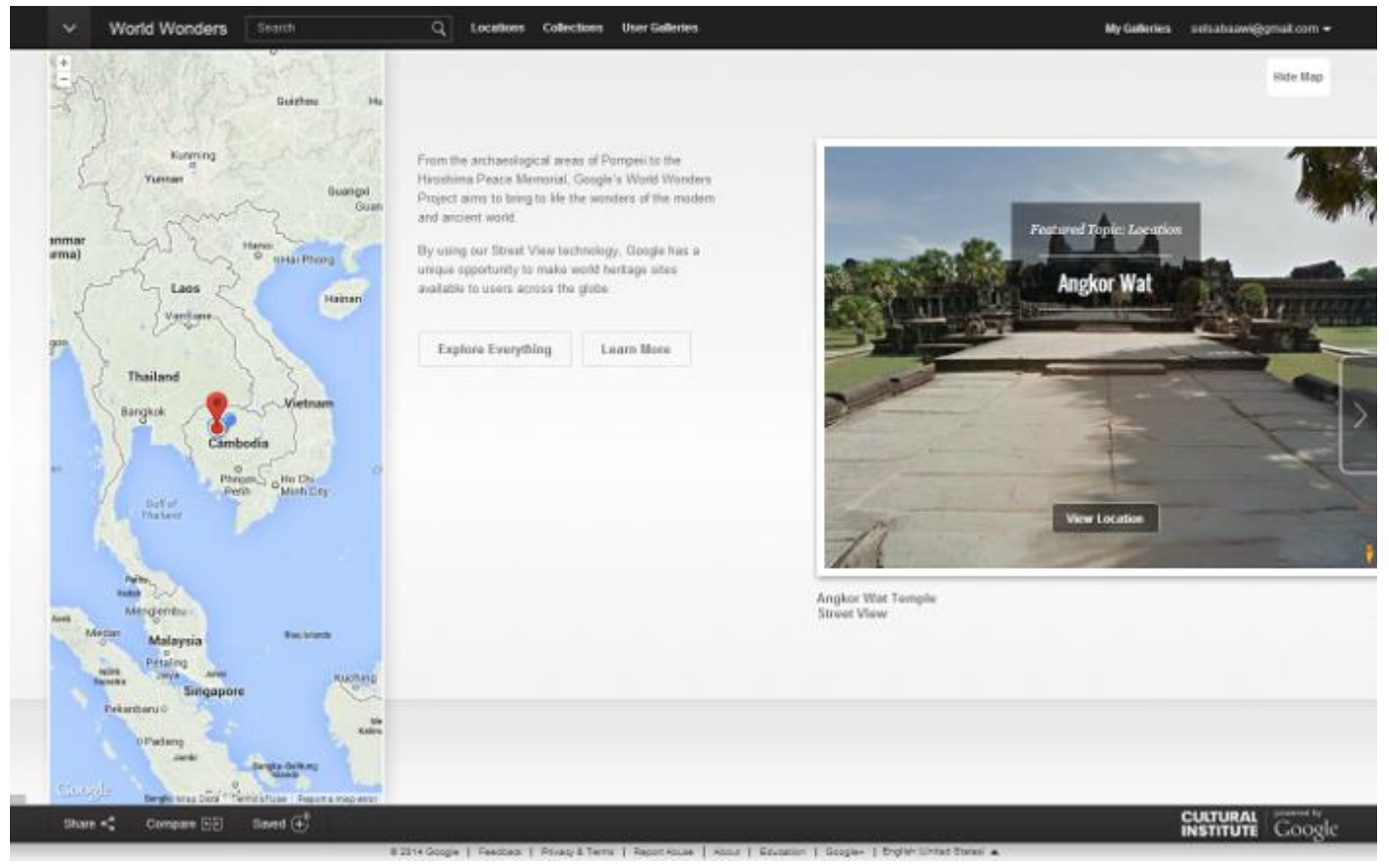

Figure B.4: A screenshot of the main page of World Wonders. Image taken by Soha ElSabaawi. 


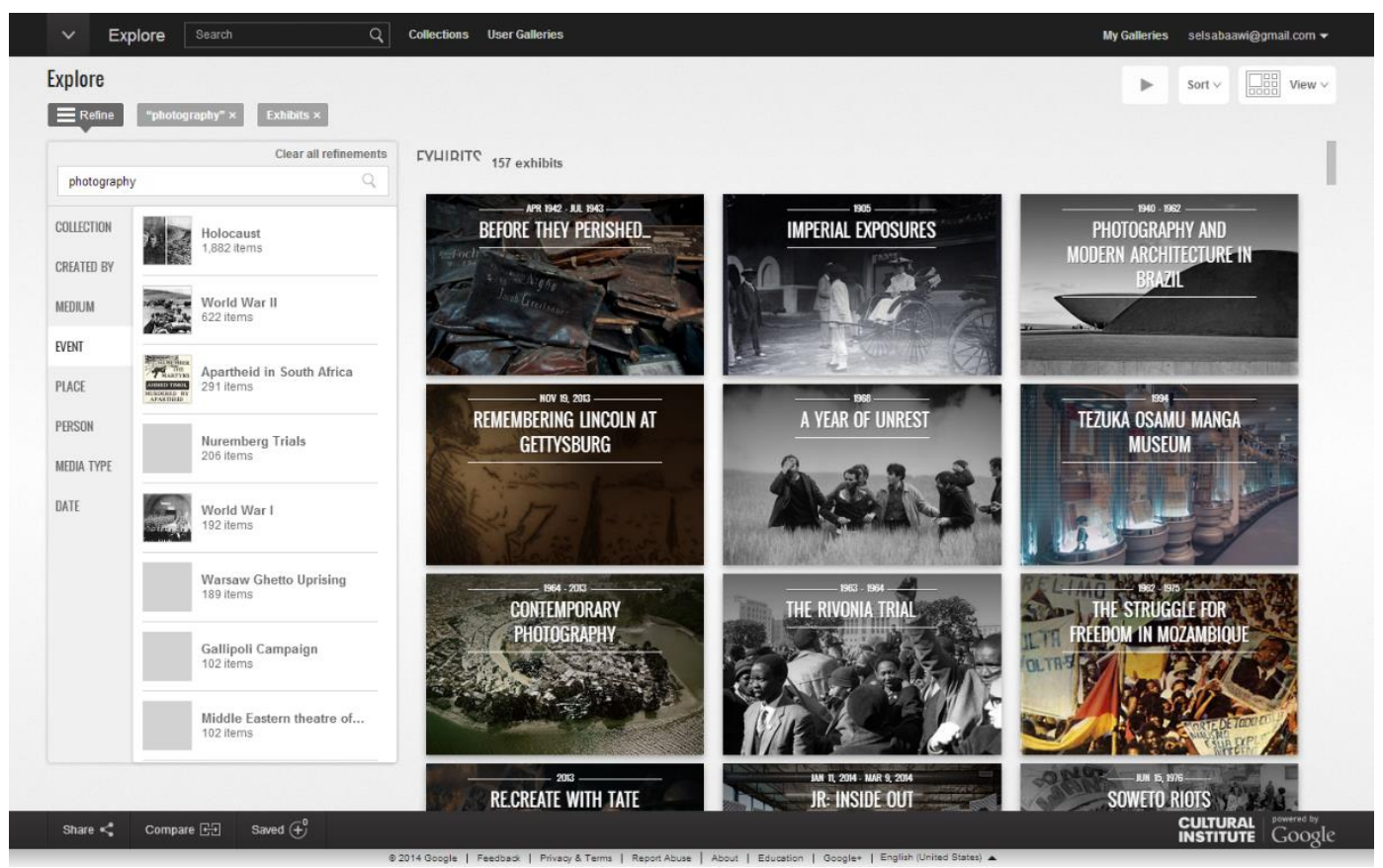

Figure B.5: A screenshot of the search results after initiating a query for "photography" and then narrowing the results down to "exhibits." Image taken by Soha El-Sabaawi.

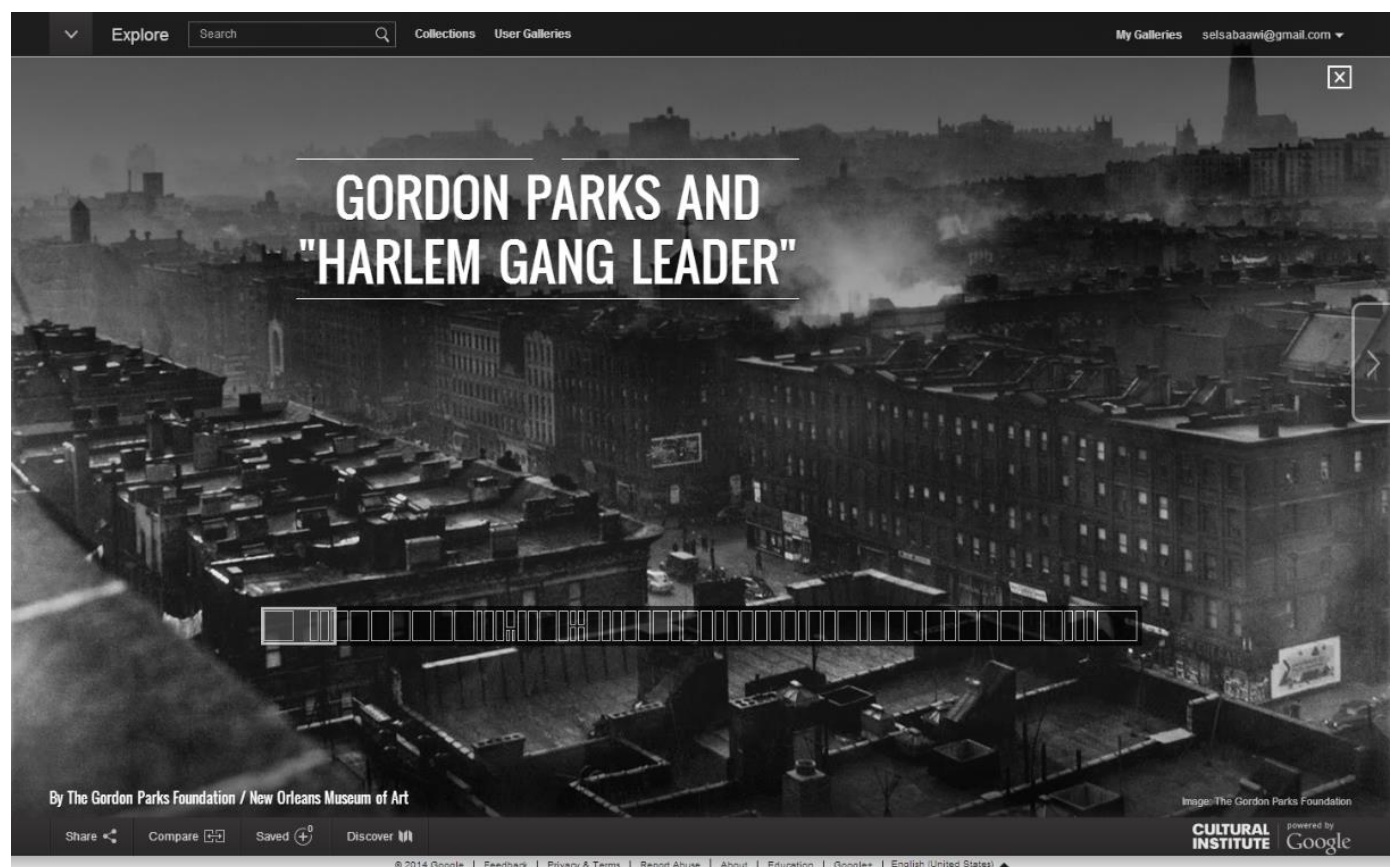

Figure B.6: A screenshot of the opening page for exhibition "Gordon Parks and 'Harlem Gang Leader." Image taken by Soha El-Sabaawi. 

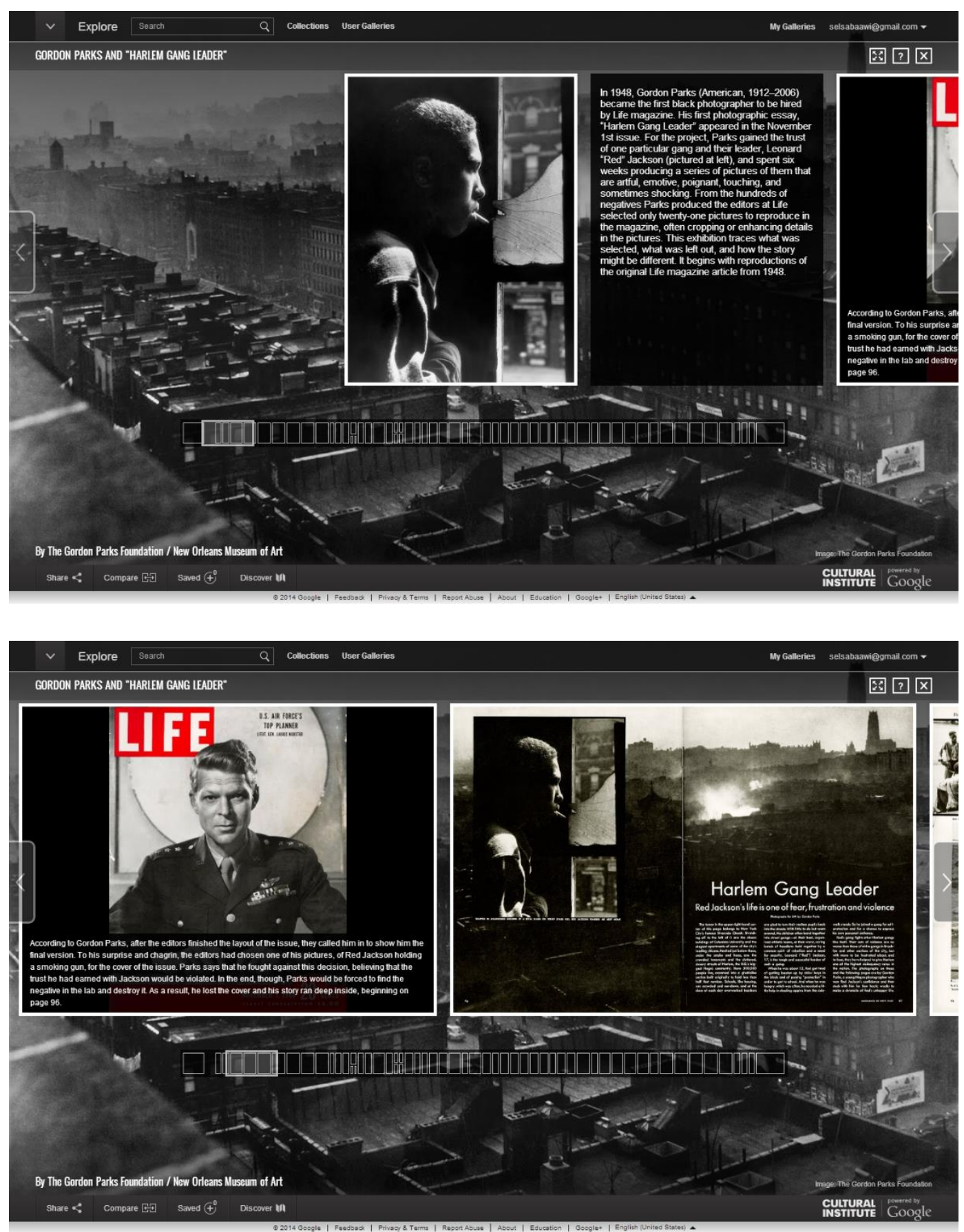

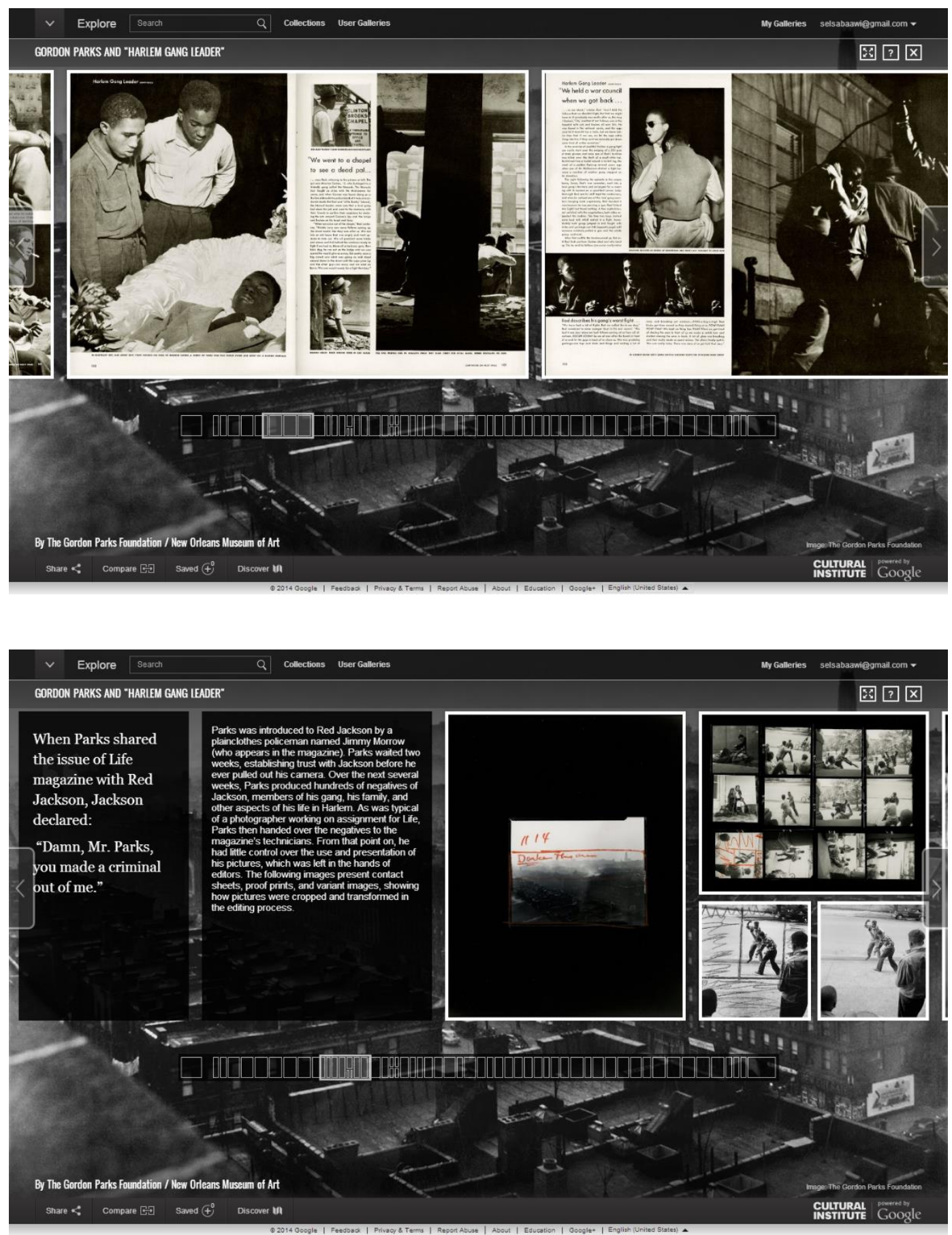

Figure B.7: The above screenshots are the first four sections to appear when scrolling through the exhibition. Images taken by Soha El-Sabaawi. 


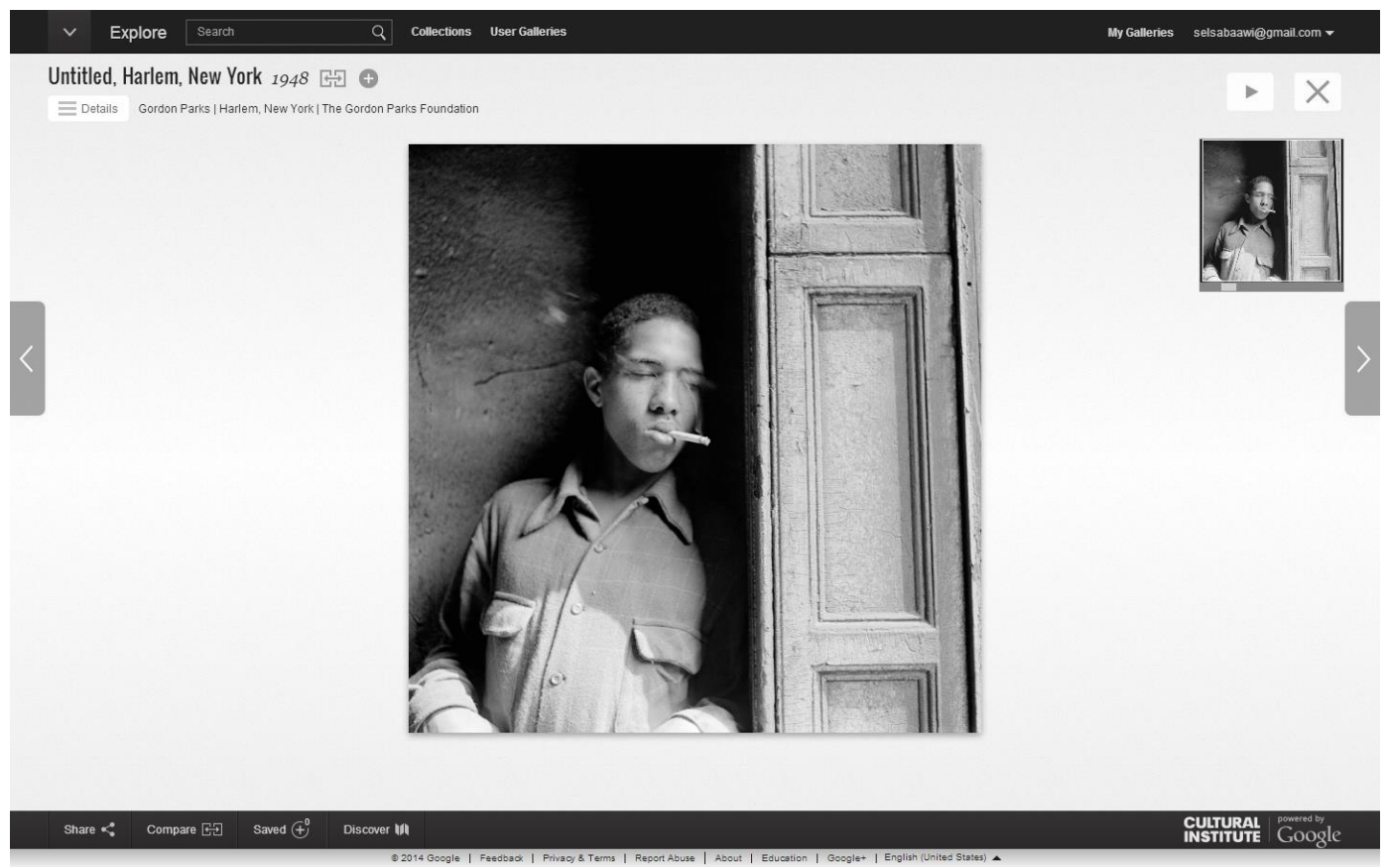

Figure B.8: A screenshot after selecting the image Untitled (1948) by Gordon Parks. Image taken by Soha El-Sabaawi.

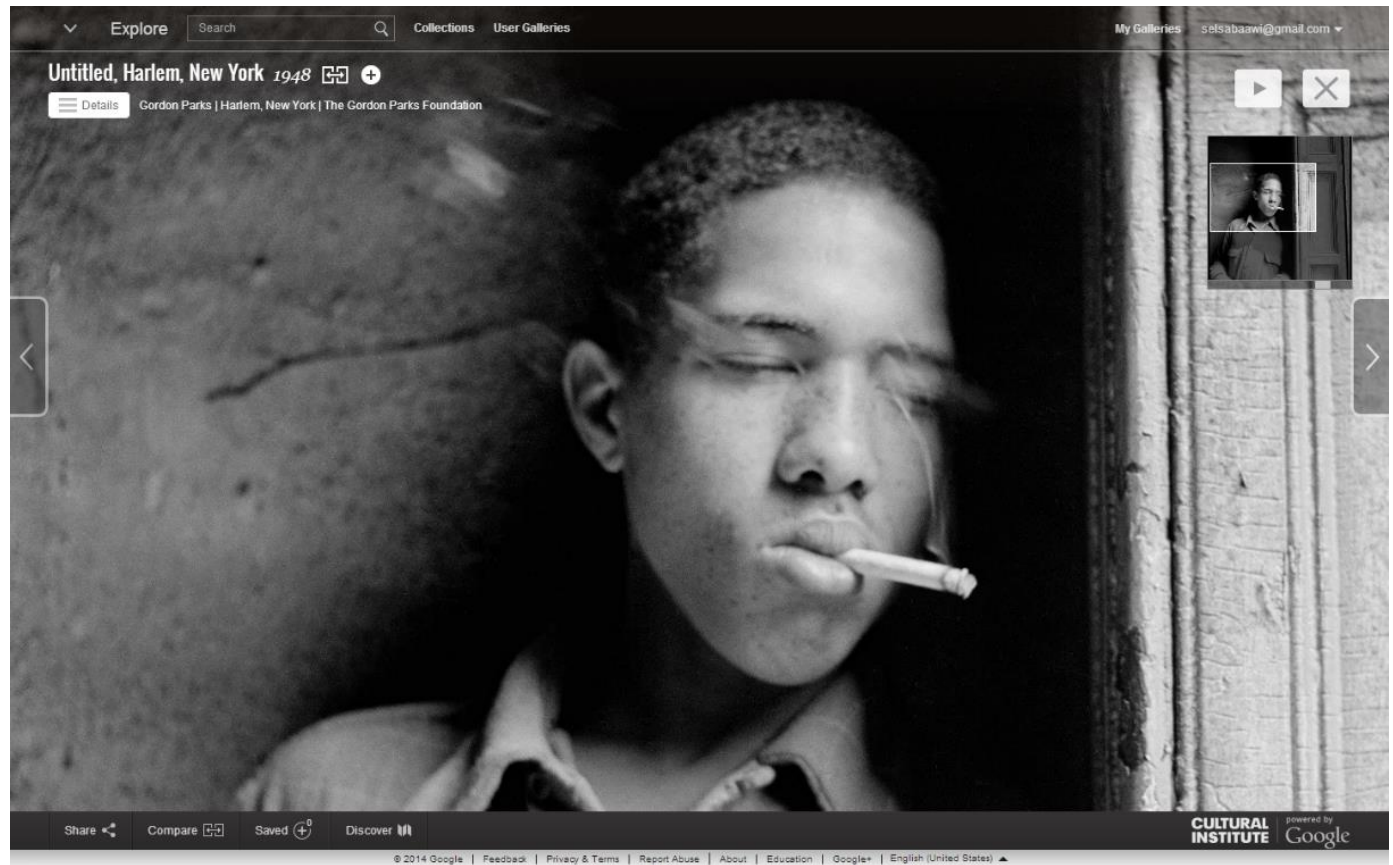




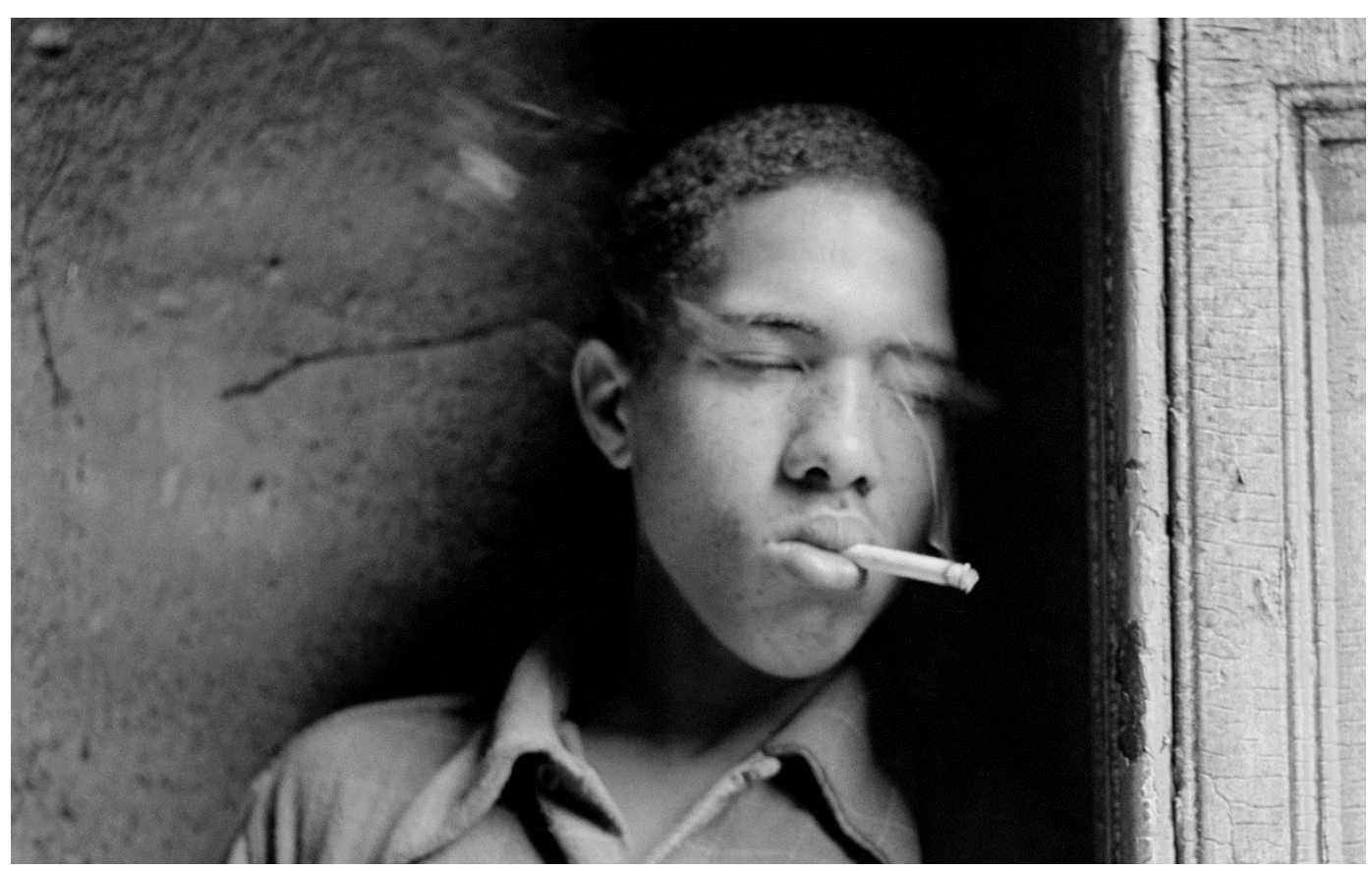

Figure B.9: A close-up of Untitled, with a user-interface display overlaying the image (top) and a close-up without it (bottom). Screenshots taken by Soha El-Sabaawi.

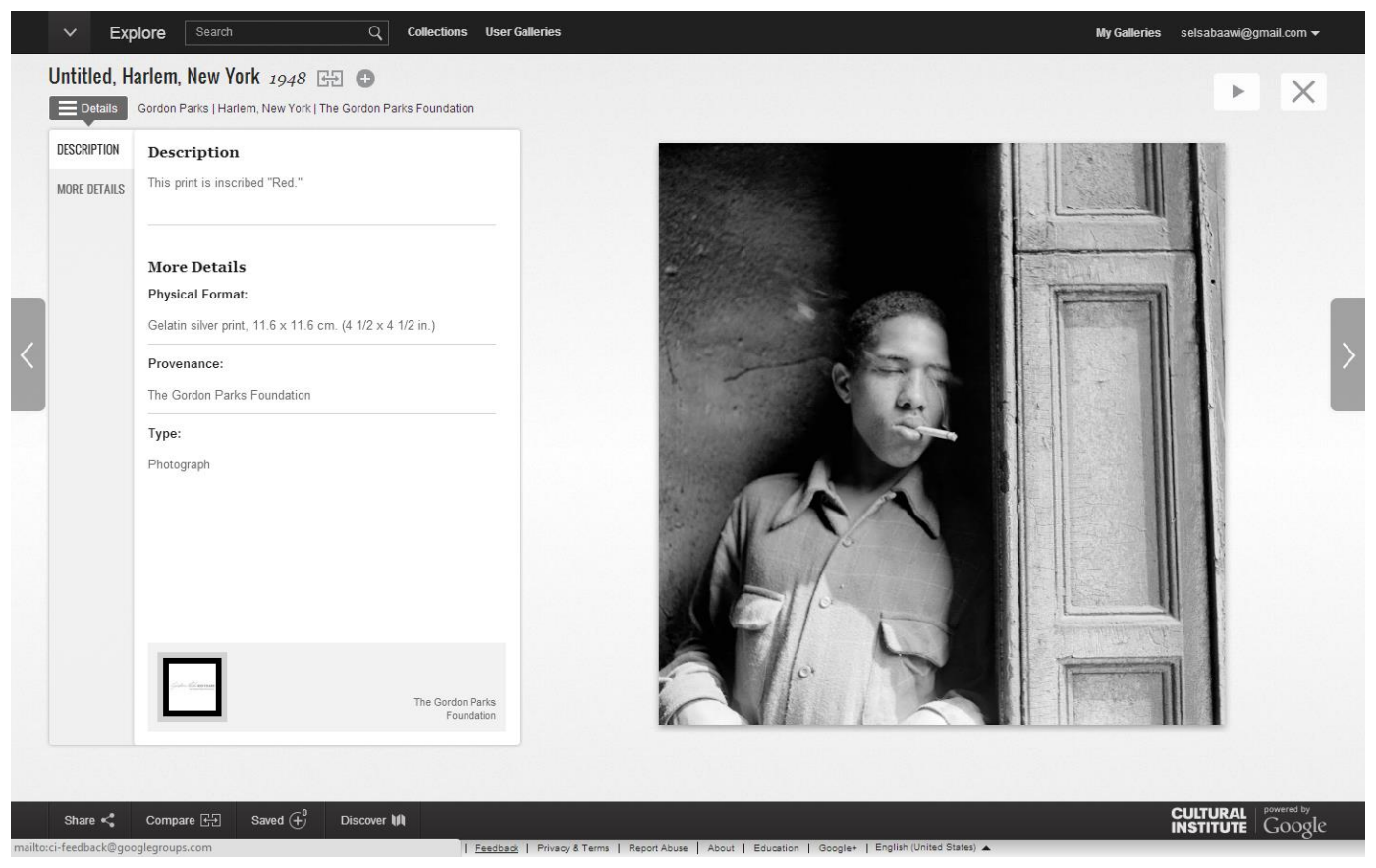

Figure B.10: A screenshot of the information pertaining to Untitled. Screenshot taken by Soha El-Sabaawi. 


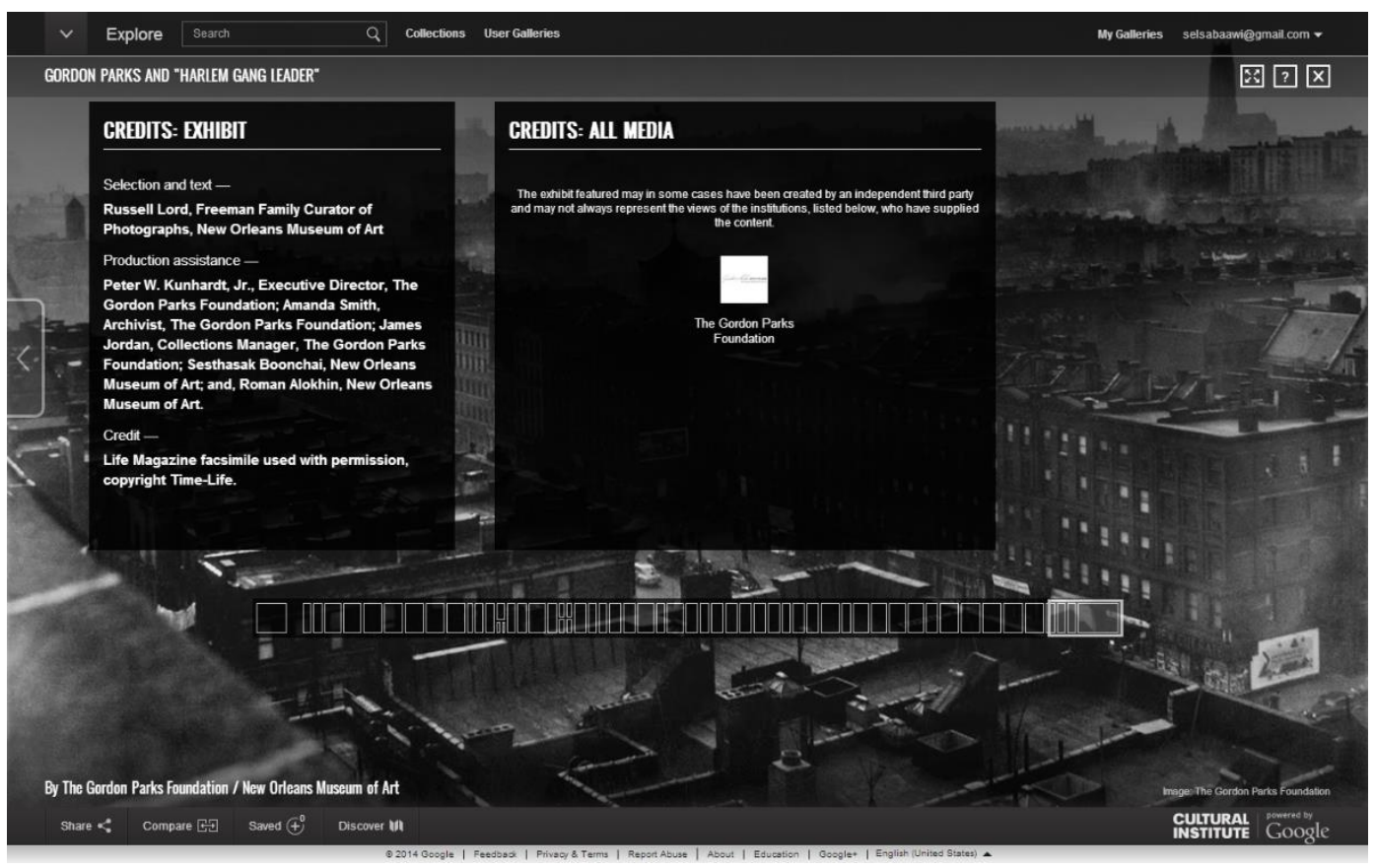

Figure B.11: The final screenshot of the Gordon Parks exhibition to acknowledge and credit those who participated in designing both the physical and virtual components. Image taken by Soha El-Sabaawi. 


\section{Case Study C: Flickr: The Commons}

Flickr is an image and video hosting and management website, and a massive online tool for managing and sharing digital photographs with a reported estimate of over 8 billion photographs from more than 87 million users, and at least 3.5 million new images uploaded from users on a daily basis. ${ }^{26}$ The feature relevant to my research is called "The Commons," (fig. C.1) a project launched in partnership with The Library of Congress in 2008 in which users have access to publicly-held photographs from 86 cultural institutions that have no known copyright restrictions. The institutions include The Smithsonian, The British Library, The George Eastman House, and the Getty Research Institute, and they agree that the photographs posted on The Commons are public domain, or are owned by the institutions that are not interested in exercising legal control or that have the legal rights to authorize others to use the online materials without restrictions. $^{27}$

The type of interaction in The Commons is entirely based on user-input. A visitor will input a subject matter into the search field, press the "Search" button, and results will generate on to a new web page. For example, I searched for "daguerreotype" and retrieved diverse results (fig. C.2). I clicked on a random result, which prompted an image titled "Unidentified Bride" by the early photographic studio Southworth \& Hawes and currently housed in the George Eastman House (fig. C.3). The second image in fig. C.3 shows the type of information that appears when users scroll further down the screen

\footnotetext{
26 "The man behind Flickr on making the service 'awesome again'," The Verge, last modified March 20, 2013, http://www.theverge.com/2013/3/20/4121574/flickr-chief-markus-spiering-talks-photos-and-marissamayer

27 "About the Rights Statement," Flickr: The Commons, accessed June 1, 2014, https://www.flickr.com/commons/usage/
} 
and away from the photograph. This includes an accession number from the respective institution's collection, maker (i.e. Southworth \& Hawes), title, date, medium, and dimensions of the original object. To the right of that information are numbers that represent the amount of times the image has been viewed, how many users have selected it as one of their favourite images, the amount of comments it has received by Flickr members, other albums it is grouped in, where else the image appears, and which words users have used to "tag" the photograph as a method of easier accessibility and searchability. The Commons not only documents where images are coming from, but also how users have responded to the images since they have been published in the engine. The language used on the website is friendly for users unfamiliar with photographic processes, and searches can be conducted by universal subjects such as "house" or "dancer," and can be tailored more specifically by utilizing an "Advanced Search" option to pick specific time frames or image files.

Museums that have partnered with Flickr for this feature have also found ways to add another layer of interactivity to showcase their collection. In April 2012, the National Maritime Museum (NMM) co-curated an exhibition titled "Curate the Collection" (fig. C.4) with seventeen selected Flickr members who chose eight images to upload onto The Commons from the NMM collection. The participating members accessed visual records of Britain's maritime history dating from the 1840s, and were taught how exhibitions are put together, such as writing wall text, constructing displays, and choosing themes through a series of workshops and discussions. ${ }^{28}$ An example of the wall text associated

\footnotetext{
28 “About Curate the Collection," Curate the Collection (blog), last modified April 16, 2012. http://curatethecollection.wordpress.com/about/
} 
with the physical exhibition is for an image titled The 'Baron Aberdare' accident (fig. C.5), which features "tags" Flickr members would use to identify and describe the image. Some of the tags operate on a sense of humour such as "Insured?" and "Oops," and are clearly using casual language to visualize the narrative and lead the conversation attached to the image. This was a unique project for NMM to undertake, as the participating users' backgrounds and knowledge in photographic history and exhibitions widely varied. Essentially, the objective of the exhibition was to move away from a standard form of exhibition and towards a narrative constructed by multiple voices and perspectives as a method of community building. Other institutions that have adopted a similar approach to exhibit their collection from Flickr members' input include the Library of Congress, the Smithsonian Institution, and the Tyne \& Wear Archives \& Museums. ${ }^{29}$

The Commons encourages a photographic understanding that is entirely based on user-input by users de-contextualizing images from a collection, and then recontextualizing the images in a different narrative. Instead of interacting with exhibitions that are already curated and labelled as witnessed from the first two case studies, this feature on Flickr is effectively utilized when users interact with the photographs to create their own, personalized meaning. The feature is used to its full potential when users are selecting, categorizing, and labelling photographs independently from an institution's standards. This case study shifts away from institutions pointing out the essence of what

\footnotetext{
29 "Making Sense of Historic Photographic Collections on Flickr The Commons: Institutional and User Perspectives," MW2013: Musems and the Web 2013, last modified January 31, 2013, http://mw2013.museumsandtheweb.com/paper/making-sense-of-historic-photographic-collections-onflickr-the-commons-institutional-and-user-perspectives/
} 
makes a photograph important, thus making the visitors of the collection valued by institutions empowering them as curators.

\section{Case Study C: Figures}

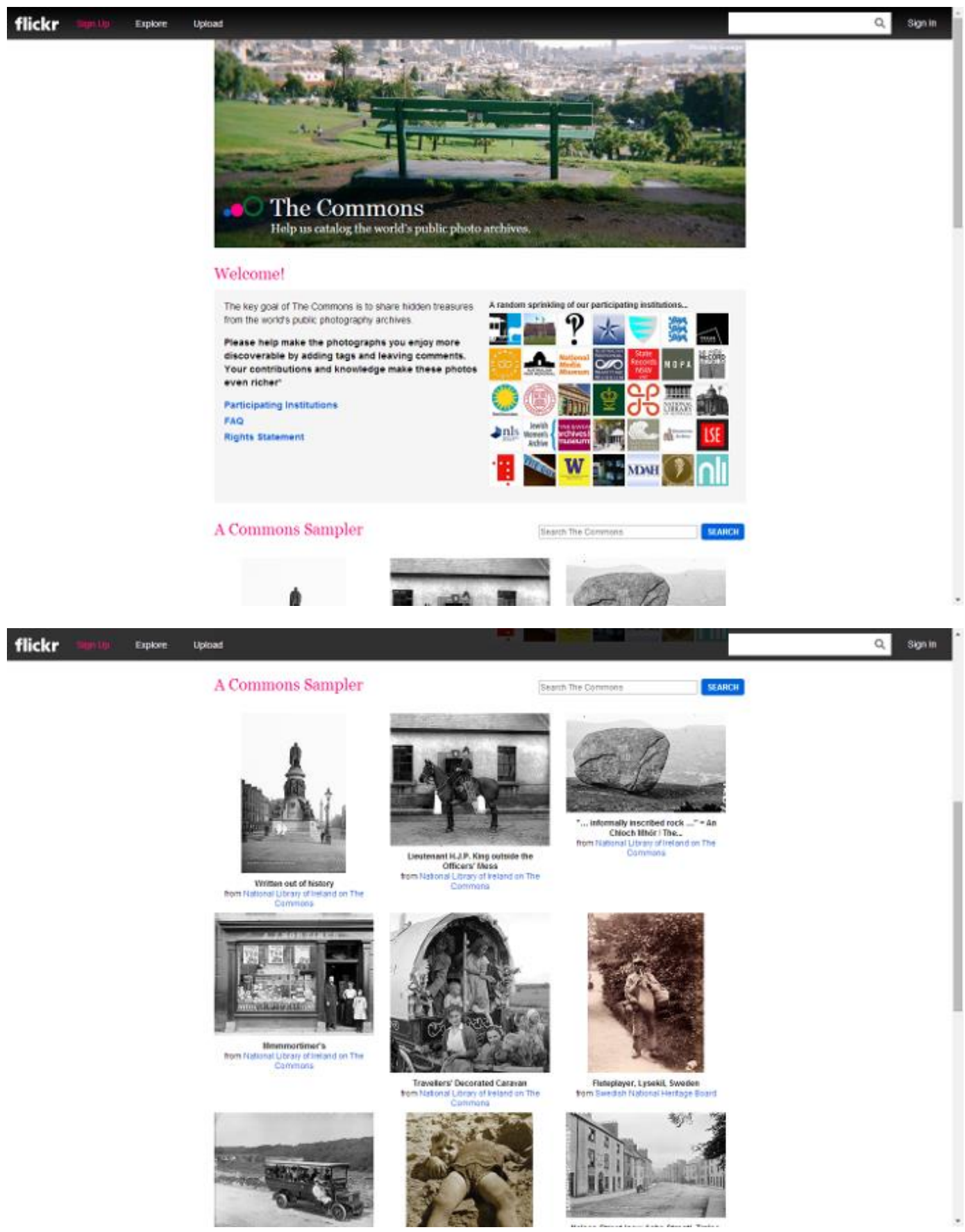


Figure C.1: Screenshots of the main page of Flickr: The Commons. Images taken by Soha El-Sabaawi.

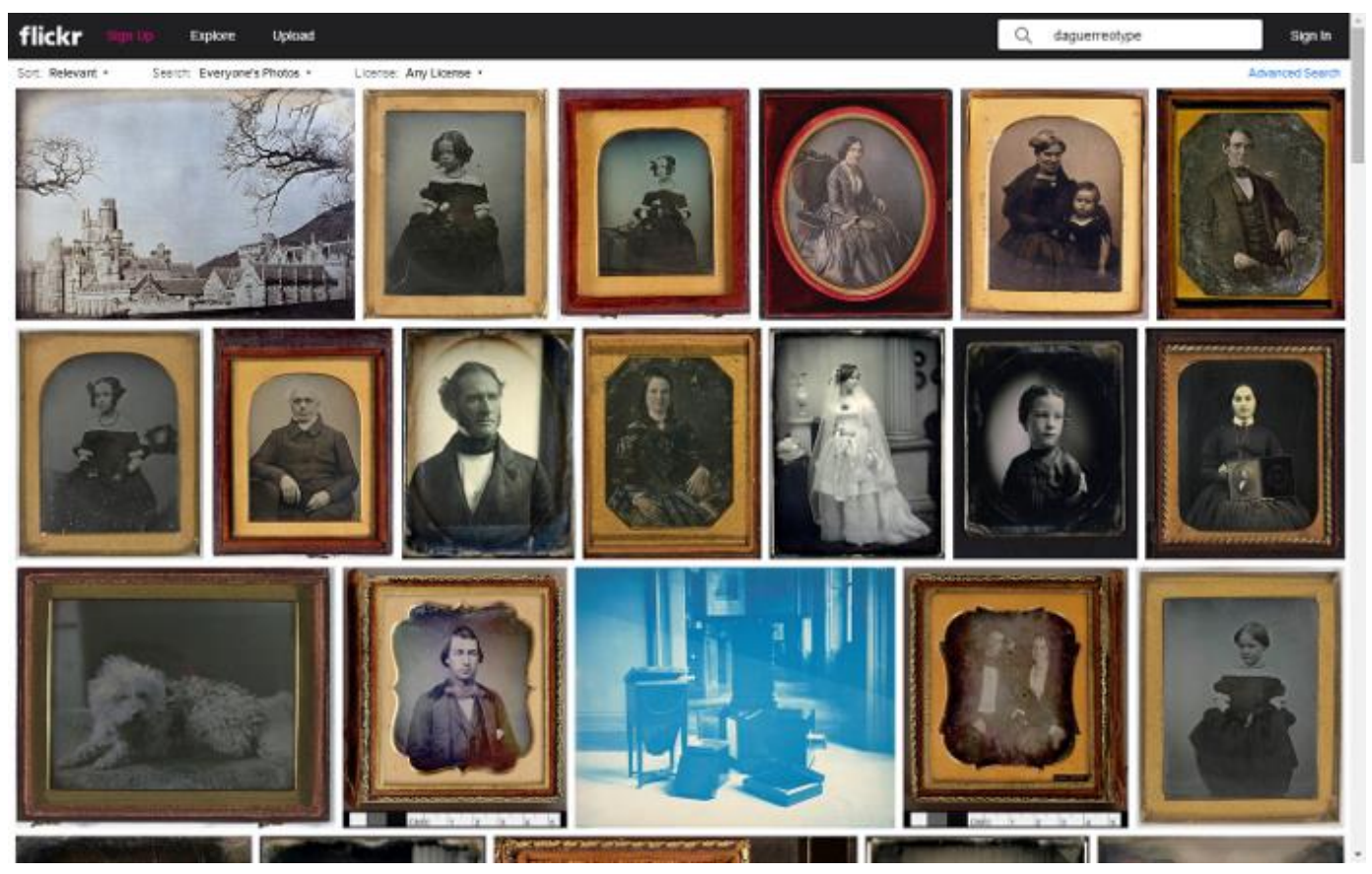

Figure C.2: Screenshot of search results for the subject "daguerreotype" in The Commons. Image taken by Soha El-Sabaawi.

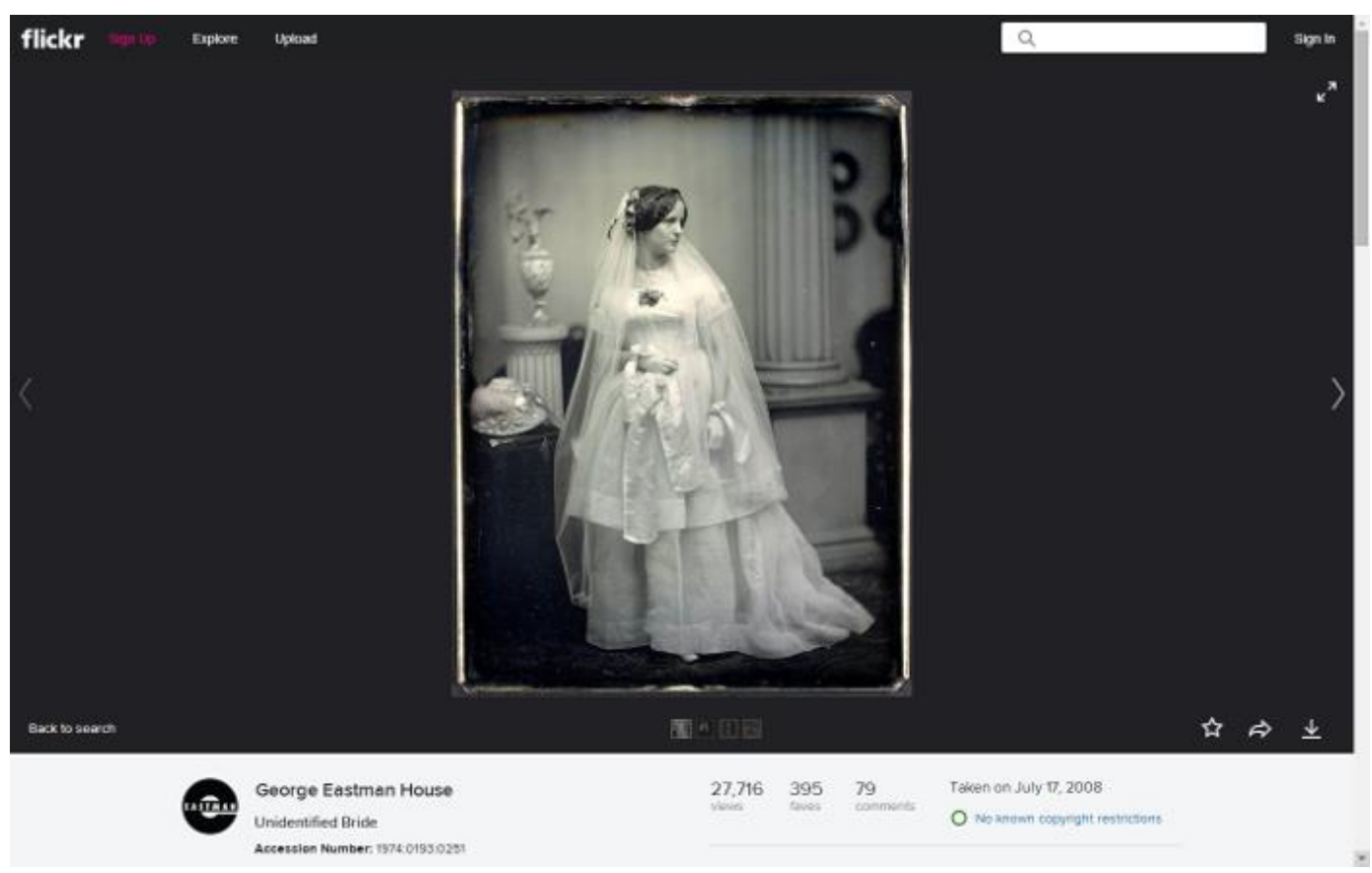




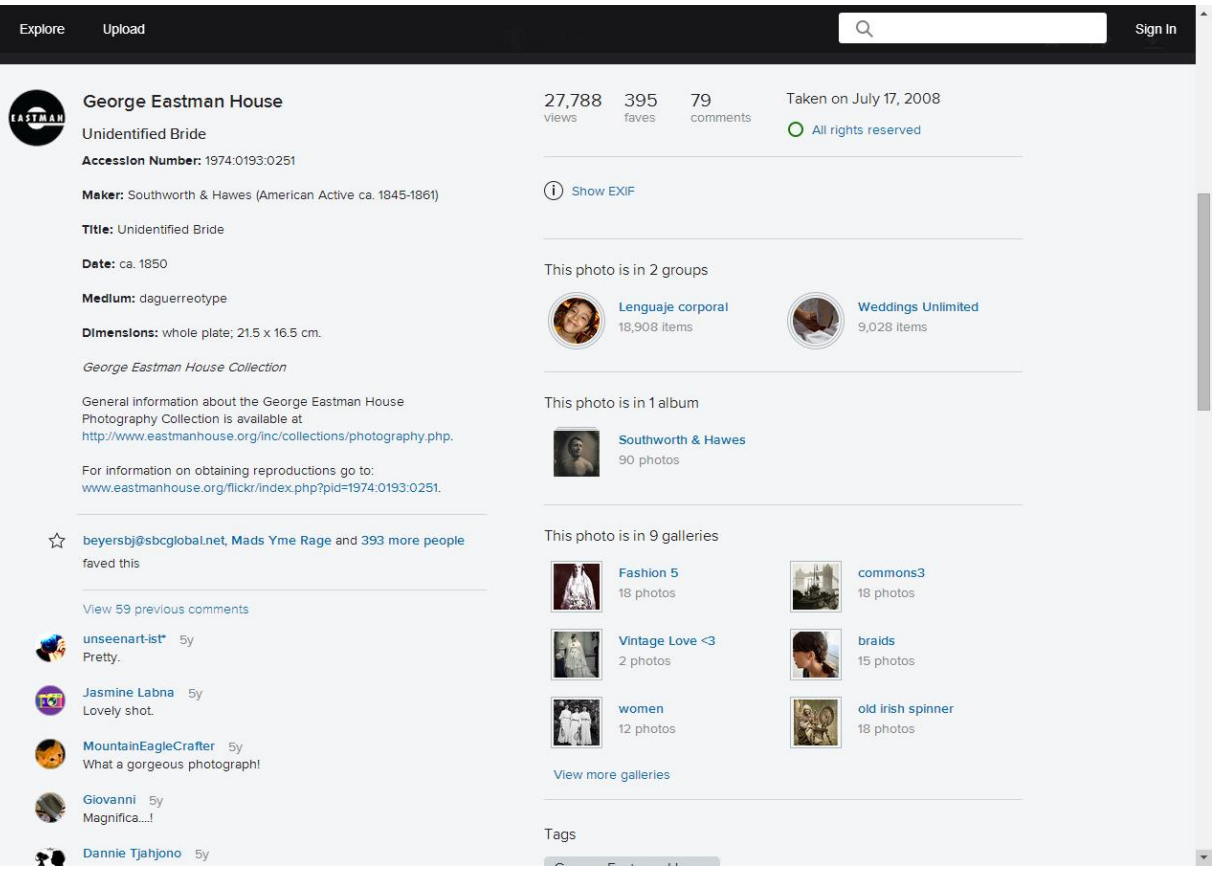

Figure C.3: Screenshots of a daguerreotype titled "Unidentified Bride." The second image is the information that appears underneath the photograph. Images taken by Soha El-Sabaawi.
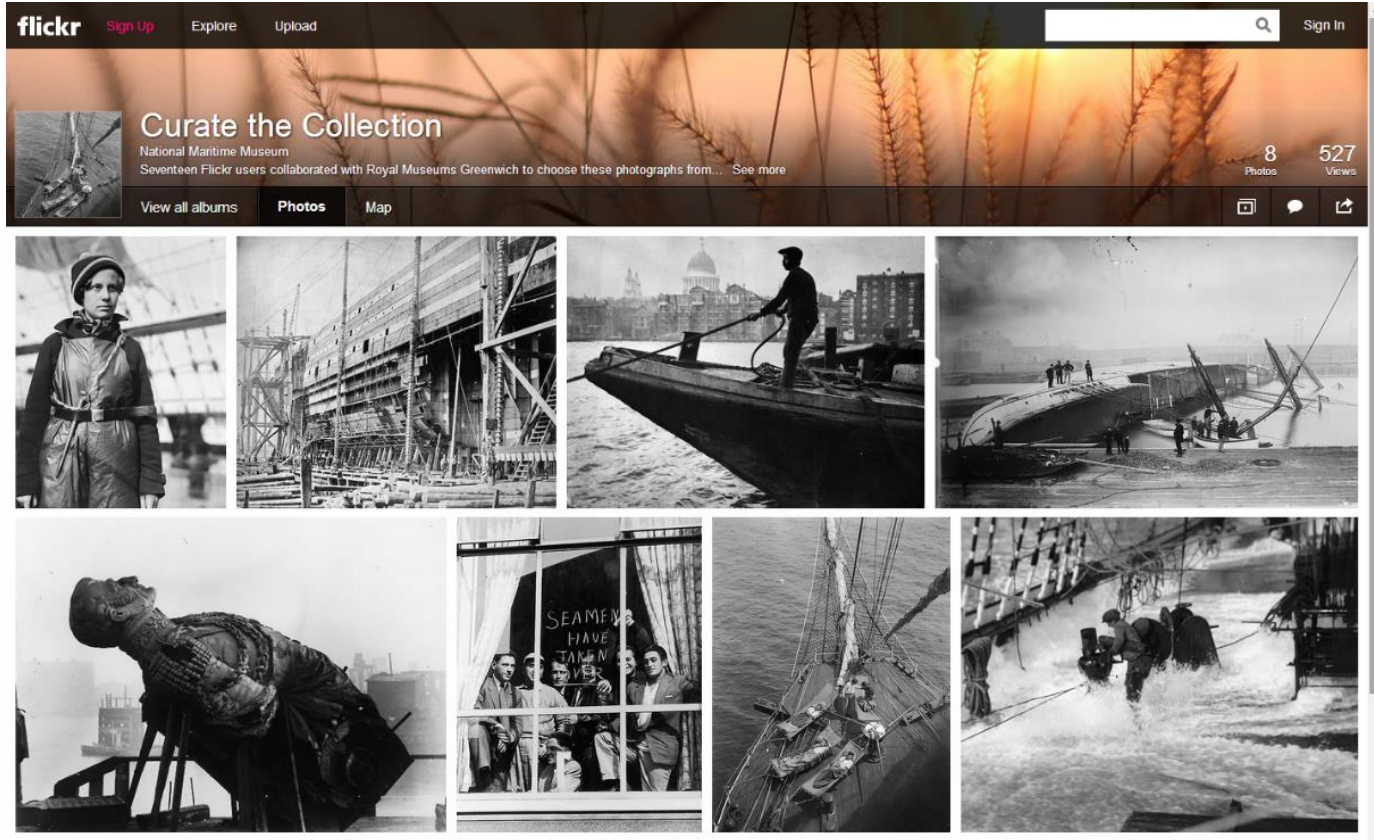

Fig C.4: A screenshot of "Curate the Collection," an online exhibition curated by the National Maritime Museum and seventeen Flickr users. Images taken by Soha ElSabaawi. 

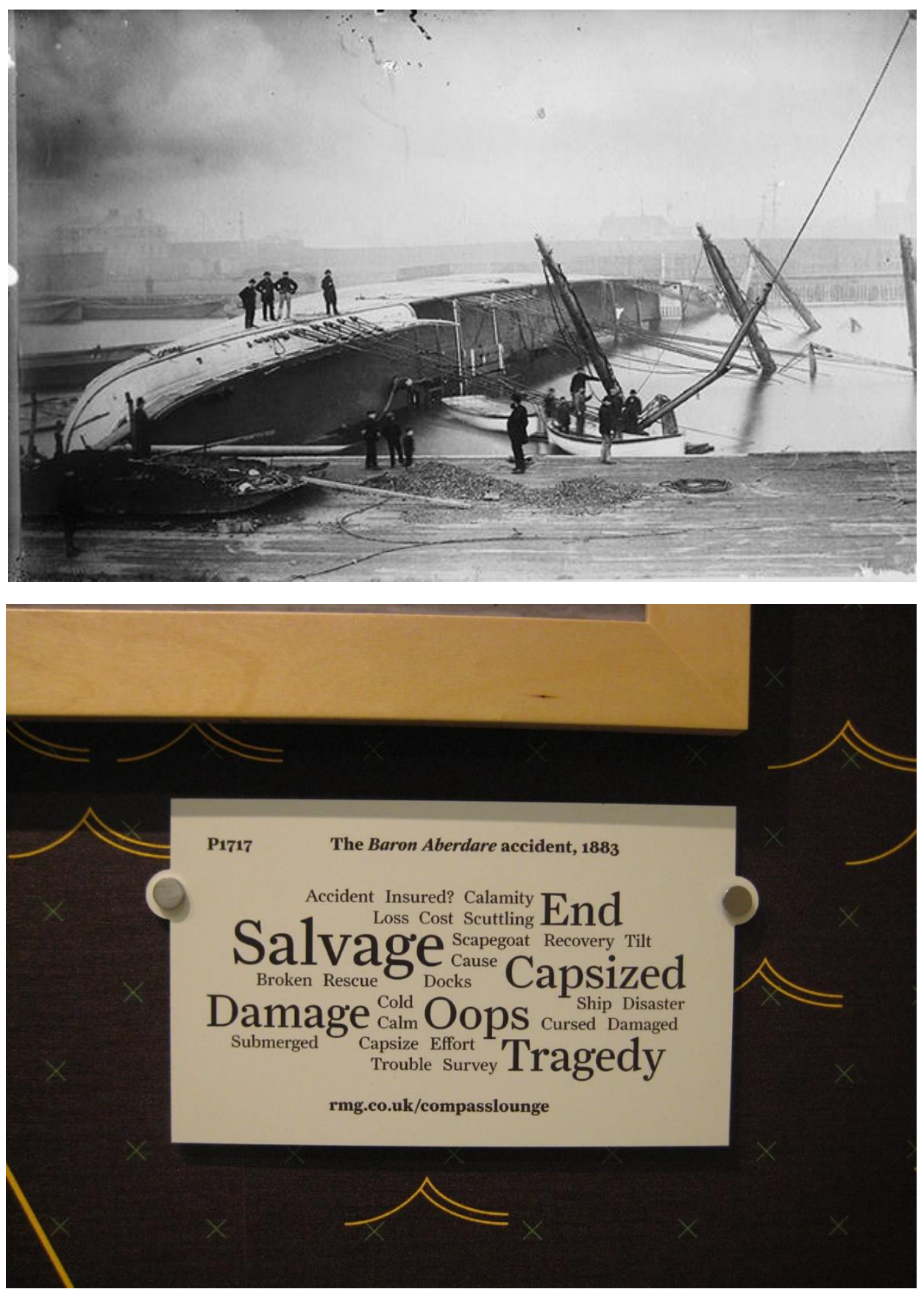

Figure C.5: Top image is a reproduction of The 'Baron Aberdare' accident (1883) courtesy of the National Maritime Museum. Bottom image is the wall text created by Flickr members in the physical installation at the NMM. Image credit: Bronwen Colquhoun and the Royal Museums Greenwich. 


\section{Case Study D: Lowcountry Digital History Initiative}

The Lowcountry Digital History Initiative (LCDHI) is a project hosted by the Lowcountry Digital Library (LCDL) at the College of Charleston in South Carolina to create a digital public history. The LCDL creates digital projects "that strengthen the College of Charleston's instructional programs and support research about the region," and are "committed to a multifaceted approach that incorporates historical and anthropological scholarship, oral history, integrative archival practices, digital librarianship, and spatial, temporal, and environmental information." ${ }^{30}$ The initiative launched in 2014 as a resource for scholars to translate archival materials into digital public history exhibitions with a major goal to encourage projects that focus on marginalized people based on race, gender, class, and labour histories. Upon visiting the project's website, users are able to look at "Exhibitions" wherein scholars have provided a historical and academic context for archival materials (fig. D.1). For this case study, I will be using “The Charleston Hospital Workers Movement, 1968-1969” (fig D.2) exhibition as an example of how archival photographs function in the space of the online initiative. The exhibition is about the "development and aftermath of the Charleston Hospital workers' strike that took place in Charleston, South Carolina, from March to July 1969."31

The introductory page of the exhibition (fig. D.3) is a description of the exhibition with a main image, and a sidebar to the left of the passage that contains the different

\footnotetext{
30 “About the Lowcountry Digital Library,” Lowcountry Digital Library, accessed June 20, 2014, http://lcdl.library.cofc.edu/about

31 "The Charleston Hospital Workers Movement, 1968-1969," Lowcountry Digital History Initiative, accessed June 20, 2014, http://ldhi.library.cofc.edu/exhibits/show/charleston_hospital_workers_mo
} 
sections visitors can view. Similar to the Google Cultural Institute, visitors can view portions of the exhibition in a linear or non-linear timeline. However, for the academic content that accompanies the archival documents, it is better to follow a linear pattern to gain the context of the full research and follow the timeline of the events surrounding the strike. There are eight sections in the exhibition: Introduction, Built on Grassroots Organizing, A Movement Gains Momentum, Civil Rights Unionism, Coretta Scott King Visits Charleston, The Settlement, Aftermath, and Sources. Most of the pages contain a narrative of the timeline of events with images to the left and right of paragraphs as seen in fig. D.4, but other archival materials utilized are documents and screenshots of film stills such as the ones in fig. D.5. From viewing the figures that I have listed so far, there are both captions underneath the photographs and documents alongside the research written in the text surrounding them. The captions function similarly to wall texts that accompany photographs in a physical exhibition. However, the main difference is that they do not include photographic information such as a credit to the photographer, the size of the actual image, or the type of photograph being presented. Instead, the caption focuses on a short descriptive title, the location of the event, and a credit to the photograph's origin before it was acquired by the initiative. Similarly, on the exhibition's "Sources" section, a credit is made to scholarly text, online resources, research centres and archives, and does not directly mention the photographs as a primary source of information. ${ }^{32}$

\footnotetext{
32 “Sources,” Lowcountry Digital History Initiative, accessed June 20, 2014, http://ldhi.library.cofc.edu/exhibits/show/charleston_hospital_workers_mo/sources_3
} 
This is an important distinction because the exhibition moves away from photography and towards a focus on social context that applies to the larger scope of the research written in the text. Bearing in mind the previous case studies, the other three consistently return to the actual object in respective exhibitions by noting cataloguing information that includes physical descriptions, and in some cases, where possible, by also crediting a photographer for the image. When exhibitions place that credit and attributes to a photograph, the materiality of the objects reminds online visitors of the fact that a photograph is an art object that has specific qualities and creators. Where the Lowcountry Digital History Initiative differs is that photographs are primarily used as a tool to accompany research and relay historic events. The images utilized are presented to relay information rather than to be treated as art objects, thereby shifting the discourse from physical materiality to social and historical context.

\section{Case Study D: Figures}
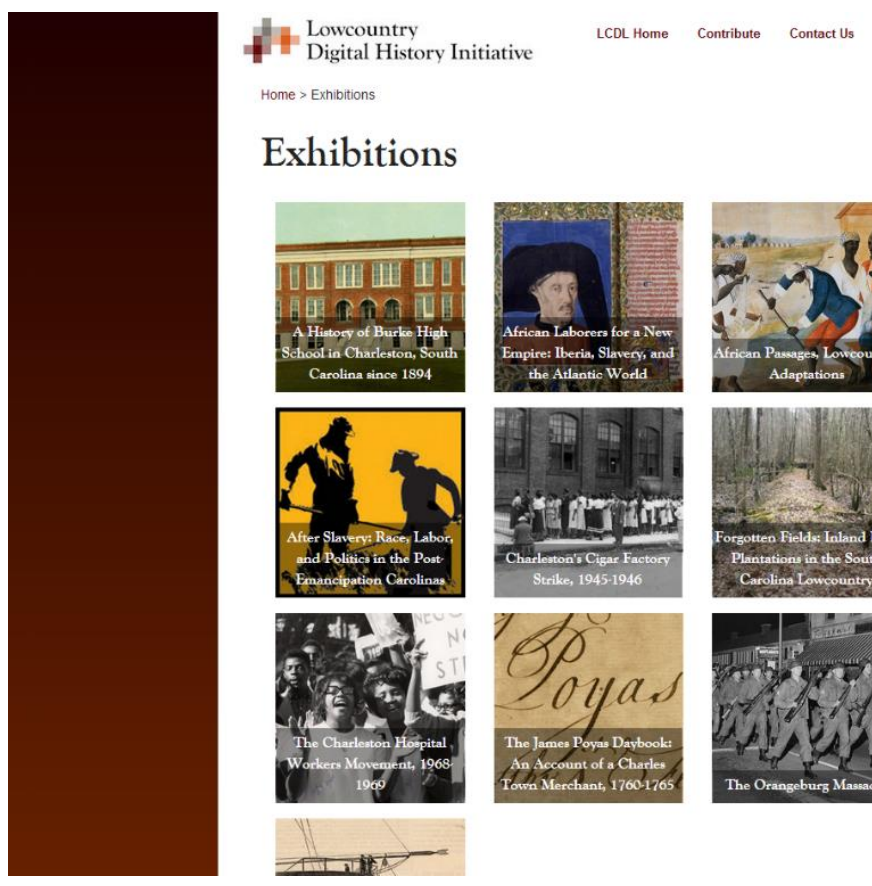

\section{Exhibitions}
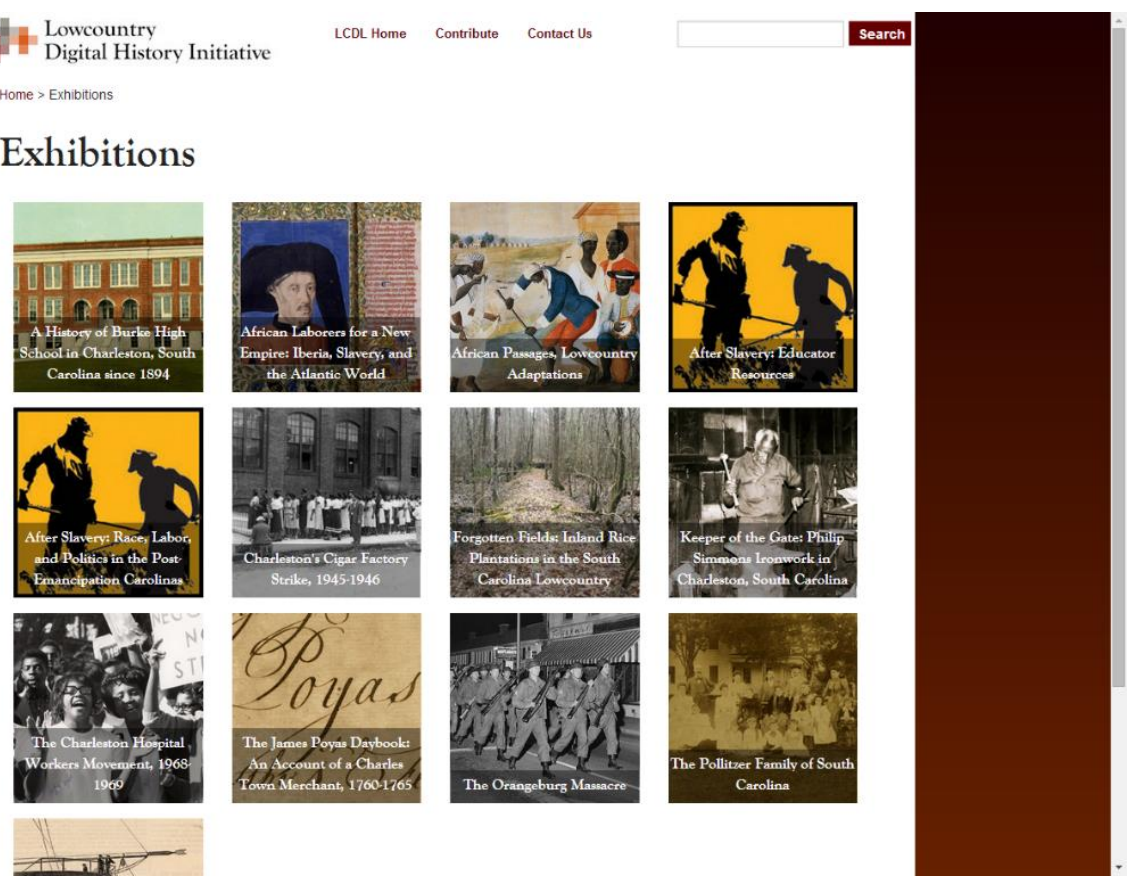
Figure D.1: A screenshot of the main collection of exhibitions for the Lowcountry Digital History Initiative. Image taken by Soha El-Sabaawi.

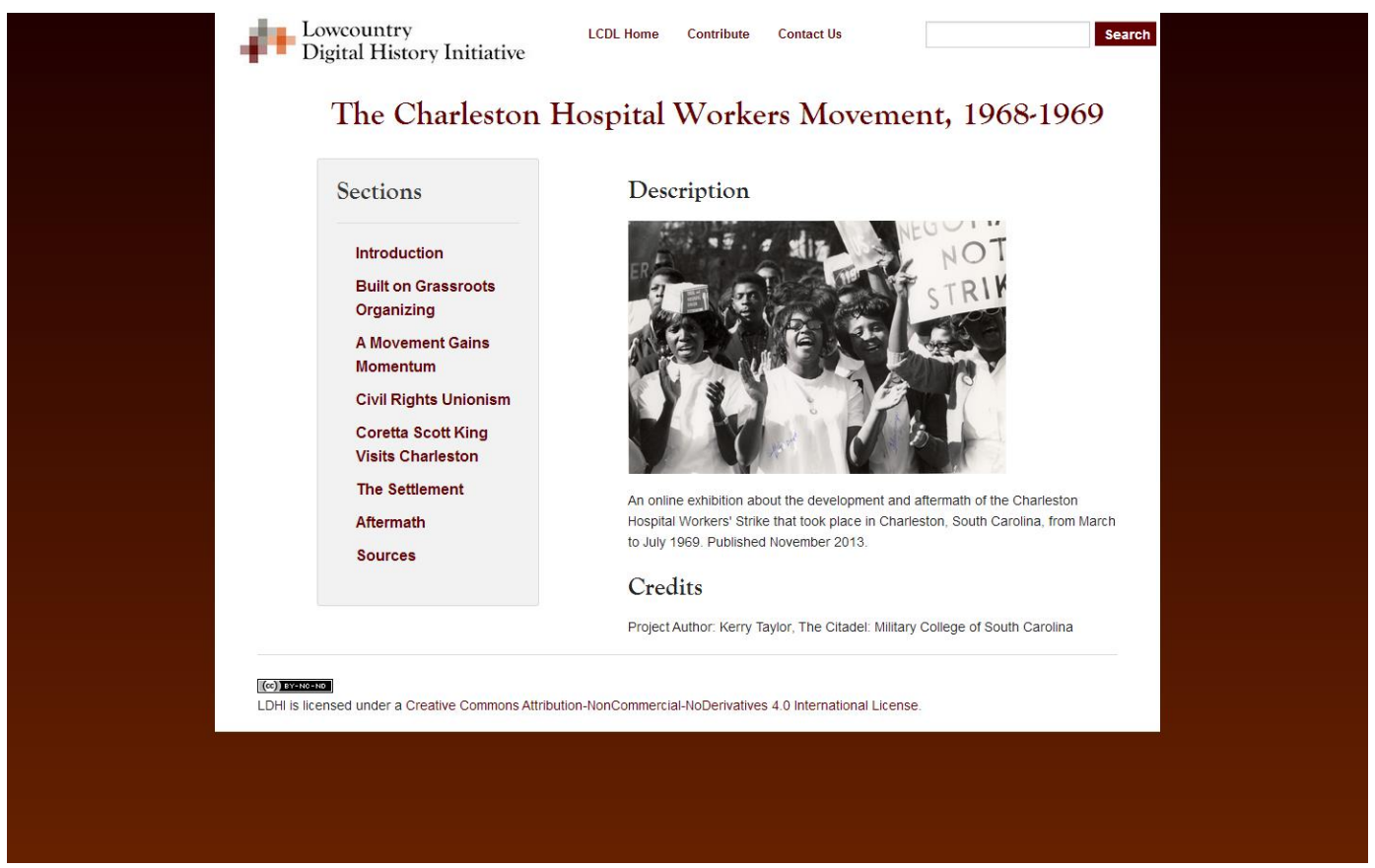

Figure D.2: A screenshot of the opening image for the exhibition "The Charleston Hospital Workers Movement, 1968-1969.” Image taken by Soha El-Sabaawi.

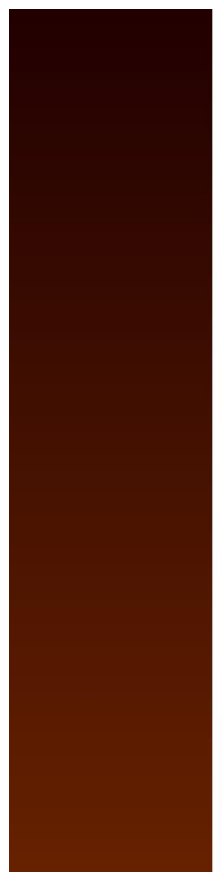

The Charleston Hospital Workers Movement, 1968-1969
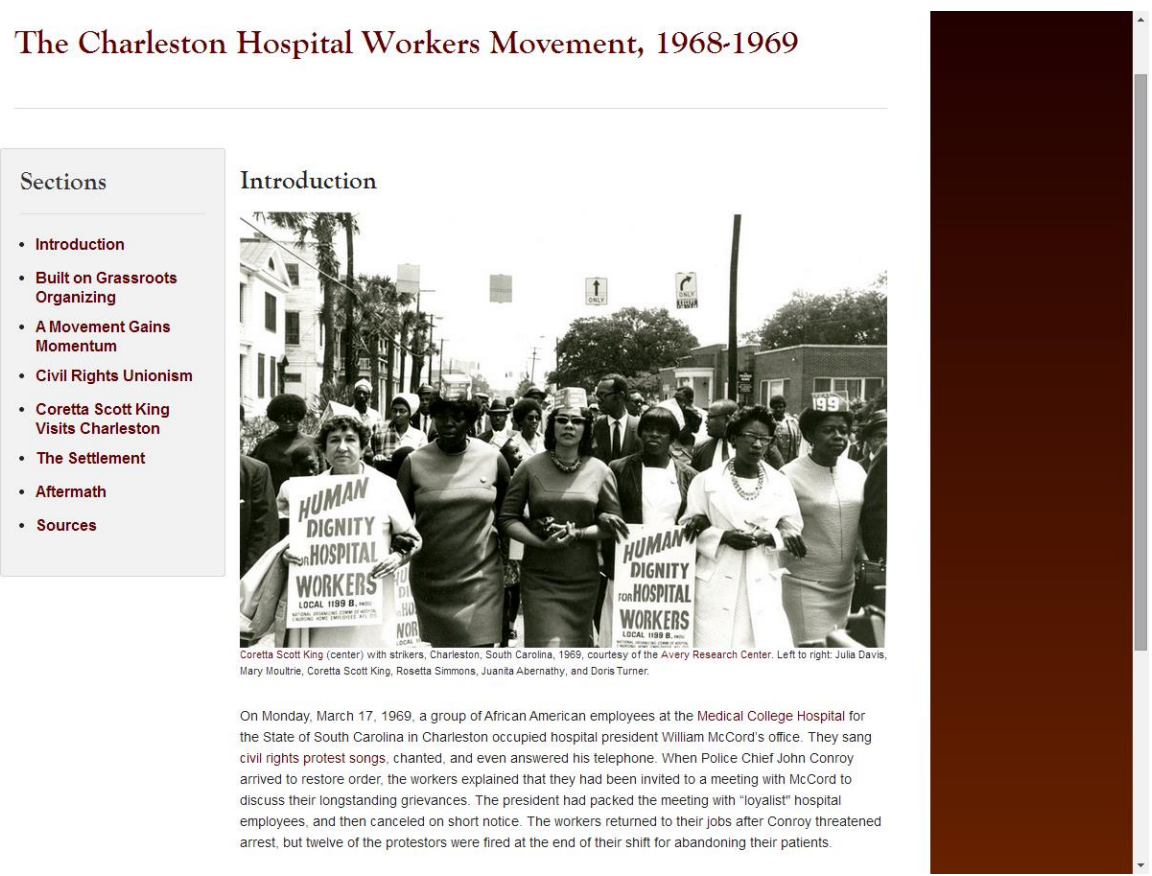

Figure D.3: A screenshot of the exhibition's introductory section. Image taken by Soha El-Sabaawi. 

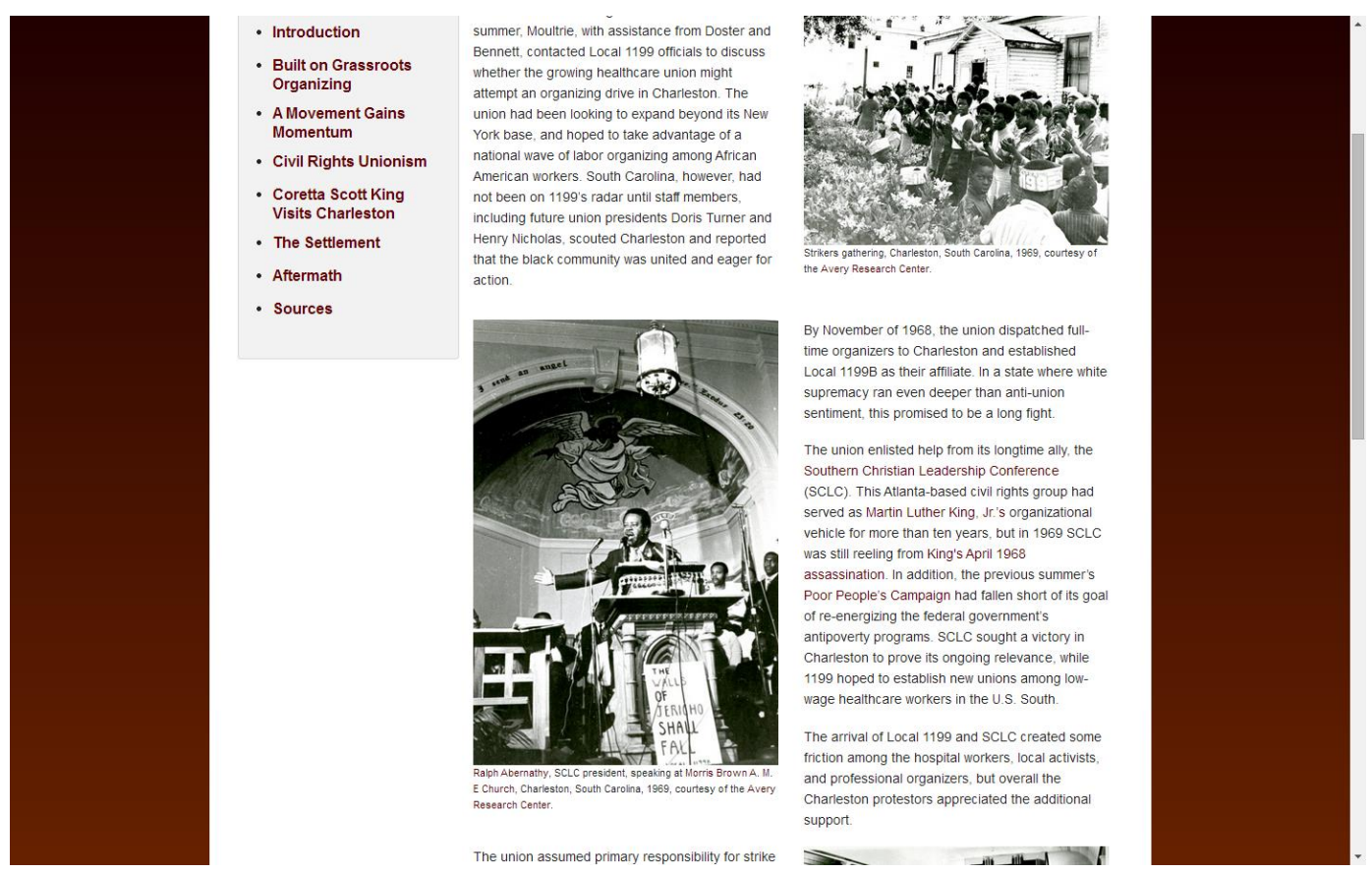

Figure D.4: A screenshot of the exhibition. Image taken by Soha El-Sabaawi.
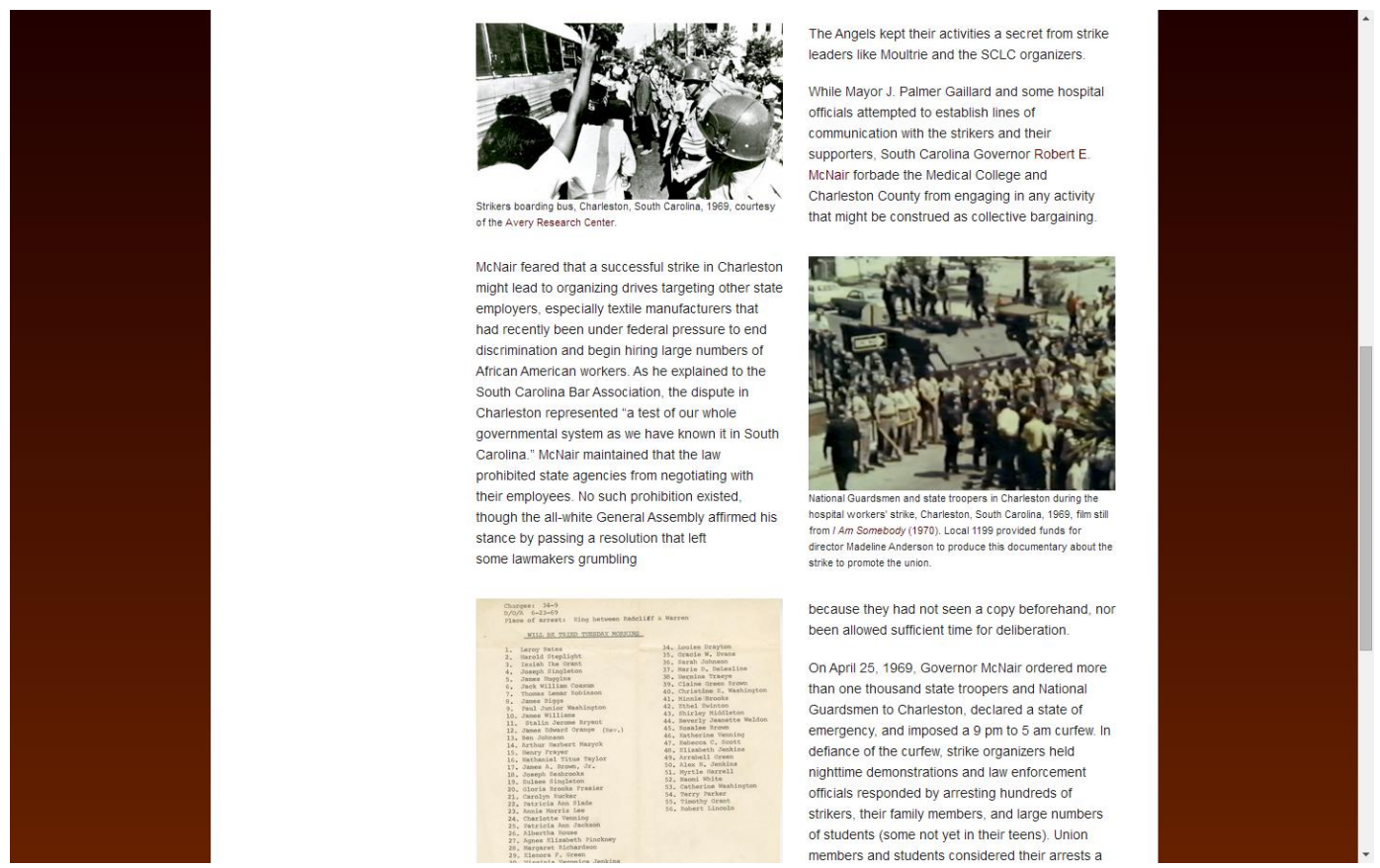

Figure D.5: A screenshot of the exhibition. Image taken by Soha El-Sabaawi. 


\section{Analysis}

The four case studies show how a digital surrogate can migrate between exhibitions, its meaning fluid and evolving to suit different narratives. What ultimately changes throughout the case studies is the architecture surrounding digital materiality, from materials appearing on a touchscreen in an institution to being available on an image database or virtual gallery and museum. Even with the changes of how images are being accessed, a message is abundantly clear: digital exhibitions call for a personalized and customizable experience based on personal, social, or historical context.

It is useful to contextualize the four case studies using insights into interface culture as found in new media studies. In Software Takes Command, Lev Manovich retraces the rise of interface culture and constructs a timeline from the 1960s to 1991 which largely credits computer scientist Allan Kay and his collaborators as spearheading the movement to make digital computers imitate older media such as newspapers, photographs, or films, using the Graphical User Interface (GUI). ${ }^{33}$ Using these "remediation machines," new media technology is consistently evolving to improve the way we access earlier media such as converting paper books into ebooks. Essentially, a user is still reading a book but experiencing the book through different kinds of media and interfaces.

The digitized, interactive exhibitions that I have examined are essentially cultural interfaces. On the topic of the interface, Manovich adds that "As distribution of all forms of culture becomes computer-based, we are increasingly 'interfacing' to predominantly cultural data - texts, photographs, films, music, virtual environments. In short, we are no 
longer interfacing to a computer but to culture encoded in digital form. ${ }^{34}$ Users are able to connect to databases and exhibitions curated by institutional professionals, which is in direct relationship to the cultural data of the institutions' collections. In PAMA, the digital interface of the mutli-touch is within the gallery space, creating access to early photographs. Google Cultural Institute is a browser-based interface that recreates a virtual experience of early and modern cultural data. As Flickr: The Commons has shown, users are even invited to recontextualize the cultural data to produce a customizable exhibition. Lastly, the Lowcountry Digital History Initiative is another browser-based interface but with an academic mandate to access historical information. The interface becomes an invisible matchmaker between what the users desire and what these institutions allow in terms of virtual spaces. Even in physical exhibitions where the collections are more limited and are still heavily influenced by the institution, there is more flexibility for visitors to decide which parts of an exhibition they prefer to access and have the ability to at the very least customize their visit to the art gallery or museum.

\section{Challenges and Potential Alternatives}

There are many advantages to utilizing interactive technology in both physical and virtual photographic exhibitions. Fragile images that cannot be exhibited in certain environments are able to be shown with a digital interface; socially marginalized groups such as low-income families and people with disabilities can access exhibitions from around the world; and digital surrogates in designated physical spaces promote more excitement from the audience and encourage them to engage with touchable tools.

\footnotetext{
${ }^{34}$ Manovich, The Language of New Media, 69-70.
} 
However, the implementation of this technology also comes with striking disadvantages and unavoidable challenges. In "Challenges of the Ubiquitous Museum," Christiane Paul discusses that striking a balance between audiences who are experienced in new media art, those who are new to it, those who have an aversion to technology, and a younger demographic more attuned to technology is "challenging, if not impossible" and lists recurring issues as a "reality check" for new media curators. ${ }^{35}$

The level of audience engagement in a physical exhibition can be difficult as those who are not comfortable with using technology will be focused on the technology itself, and not the photograph displayed within it. Paul explains that "this complaint about technology expresses frustration with its gratuitous use ... [and] this critique is linked to a person's familiarity with the medium." ${ }^{36}$ On the one hand, unfamiliar museum and art gallery visitors will more likely be intimidated by the usage of the interactive display rather than focus on how the design is being used to present photographic materials, which disrupts the purpose of going to exhibitions to contemplate art objects in person. For example, the multi-touch table located at PAMA is large enough to be off-putting for inexperienced visitors who would rather have the original object across from them. Installing more digital technology in physical spaces will not likely train inexperienced visitors to use them more, as they will more likely avoid the exhibitions that utilize that technology altogether, and move towards a traditionally designed show. Similarly, those "visiting" virtual galleries like Google Cultural Institute or Flickr: The Commons for the first time will initially need to adjust to the designs' features in order to comfortably

35 "Challenges for a Ubiquitous Museum," 66.

${ }^{36}$ Ibid., 67. 
navigate them as they are cluttered with images and minimal text with little direction, which can be frustrating and disengaging. However, the Lowcountry Digital History Initiative has a cleaner interface, but is likely to be more engaging to the target audience of history scholars based on its content. On the other hand, visitors who are experienced in interacting with interfaces and touchscreens will be able to adapt and appreciate the designs much quicker, and pick up on the social cues integrated within the websites like sharing, tagging, and searching for photographs because these are common terms used across most present social media networks. An alternative solution for this problem would be to include a digital component within a physical exhibition as a complementary tool, such as displaying the original object in a case beside a tablet showing the different views of the image. In virtual spaces, the best alternative would be to have a visible guide across the screen that acts as a tutorial for users visiting the space for the first time.

Another concern that Paul points out is that art audiences play the role of "art consumers" who have a desire to look at art, and "art that breaks the conventions of contemplation and purely private engagement shocks the average museumgoer, disrupting the mind-set that art institutions so carefully cultivated." ${ }^{, 37}$ Each case study can be viewed as obstructing the object for those accustomed to a contemplative art experience due to the focus on the exhibition's design. This especially concerns art institutions that display paintings and sculptures alongside photography, where interactive digital surrogates are not usually present, in comparison to science and technology institutions that are much more celebratory of new media installations. It is also unlikely that visitors who prefer physical objects over technology would spend a

\footnotetext{
${ }^{37}$ Ibid., 71.
} 
large amount of time visiting virtual galleries to look at the art, unless the features of creating their own exhibitions was a personally intriguing aspect. A potential solution for this issue would simply be additional information to the hesitant visitors. In a physical exhibition, a section of wall text beside the digitized display could explain why the objects are digitized to shift the focus back onto the objects. In virtual spaces, particularly for newcomers to the website, wall text can be translated into a "pop-up" on the screen to explain why certain functions have been implemented and how they help preserve the art.

A third disadvantage that would impact the audience's experiences is if the visitors who are not used to using technology beyond the cell phone, the Internet, or GPS viewed the interactive exhibitions as a toy for children in the museum space. While interactive installations are well suited for children and young adults, an exhibition geared towards adults could be deemed infantilizing, and the overall purpose of the exhibition would be lost. If the audience views the technology as a toy when its main use is to communicate an event or act as a method of digital preservation, the impact of the collection is devalued, which hinders both the institution and the audience members. Similar to the previous alternative solution, the key is placing a viewable description that emphasizes the importance of the technology so that it is appreciated, and the initial "shock" of newcomers seeing this technology will ease into moving away from trying to understand the design and toward learning more about the material objects.

The three major disadvantages are serious concerns that curators have to try to balance if they choose to implement digital surrogates in art-focused exhibitions in order to maximize the numerous benefits that accompany them. In a perfect scenario, a curator would have to make the technology appear seamless between user and the material 
objects so that the experiences can still focus on the contemplation of photographs with fewer disruptions, while also being able to appeal to multiple demographics and levels of experience.

\section{Conclusion}

The installation of digital surrogates and interactive exhibitions is not a new phenomenon, but an increasing recurrence in art galleries and museums worldwide, shifting art institutions from static architectures of preservation to centres of evolving media and ideas. Archivists, curators, and traditional museum-goers should focus on the ways in which technology can assist in preserving the sanctity of collections and the contemplative high art experience. The research that I have presented in this paper is merely the tip of the virtual iceberg with regard to how interactive installations can change the relationship between visitors and digitized materials and their own contextualization of photographic meaning.

In placing the context of digitized photographic materials in new media studies and digital museum studies, photo historians will be able to view new technology as multifaceted methods of remediating older materials to increase the access and enjoyment

of photo collections that cannot be exhibited physically due to environmental factors. The case studies presented are examples of how diverse in-house digital exhibitions and online virtual exhibitions can present physically sensitive but culturally relevant objects. The multi-touch table at PAMA is an example of how art galleries and museums are utilizing interactive exhibitions within their own spaces. Having the ability to touch, swipe, and zoom digitized surrogates of photographic objects from the late 1800s is both appealing and educational to younger demographics and visitors fluent in the latest 
technology. Google Cultural Institute presents virtual exhibitions from institutions across the world, increasing the accessibility to famous paintings, photographs, and architecture for low-income or physically impaired demographics. Flickr: The Commons displays a nostalgic database for photography aficionados who are interested in recontextualizing the images from the database into a different medium due to the lack of copyright restrictions, mediating both the old image and newer conceptualizations of it. Finally, the Lowcountry Digital History Initiative is a scholarly resource in the form of virtual exhibitions, merging archival photographs of relevant historic events with researched text to place the documents in a linear social context, as well as casting light on the stories of socially marginalized demographics.

In conclusion, these four case studies demonstrate the growing emphasis on digital materiality as an unavoidable priority in discussing photographic literacy. The relationship between the materials and users is an instantaneous response that requires an ongoing review of how people read photographs and view them in exhibitions. Institutions would be wise to take advantage of this relationship to study what inspires visitors to immerse themselves in photographic collections. Moving forward, researchers can continue to address how striking a balance between tech-savvy and inexperienced visitors can create the most fulfilling methods of participation so that the technology becomes invisible and seamless, and the focus returns to a visitor's appreciation and contemplation of older photographic materials. 


\section{Works Cited}

Abel, Kris. "iPad App Brings Ultimate Dinosaurs To Life At The ROM.” YouTube video, 3:08. Posted June 14, 2012. https://www.youtube.com/watch?v=FhLMNGy_PX8 Baker, Natasha. "Dinosaurs Roar to Life with Museum's Augmented Reality App.” The Globe and Mail. Last modified July 16, 2012. http://www.theglobeandmail.com/technology/tech-news/dinosaurs-roar-to-lifewith-museums-augmented-reality-app/article4420174/

Bandelli, Andrea. "Virtual Spaces and Museums." In Museums in a Digital Age, edited by Ross Parry, 148-152. London: Routledge, 2010.

Bush, Vannevar. “As We May Think.” In The New Media Reader edited by Noah Wardrip-Fruin and Nick Montford, 37-48. Cambridge: MIT Press, 2003. . "Memex Revisited.” In New Media, Old Media: A History and Theory

Reader, edited by Wendy Hui Kyong Chun and Thomas Keenan, 85-95. London: Routledge, 2006.

CBC News. "Luminato, AGO Face Cuts in Ontario Budget." CBC News. Last modified March 28, 2012. http://www.cbc.ca/news/arts/luminato-ago-face-cuts-in-ontariobudget-1.1230120

Colquhoun, Bronwen. "Making Sense of Historic Photographic Collections on Flickr The Commons: Institutional and User Perspectives.” Museums and the Web 2013. Last modified January, 31, 2013. http://mw2013.museumsandtheweb.com/paper/making-sense-of-historicphotographic-collections-on-flickr-the-commons-institutional-and-userperspectives/

Coulter-Smith, Elizabeth and Graham Coulter-Smith. "Mapping Outside the Frame: 
Interactive and Locative Art Environments." In Digital Visual Culture: Theory and Practice, edited by Anna Bentkowska-Kafel, Trish Cashen, and Hazel Gardiner, 49-65. Bristol: Intellect Books, 2009.

Curate the Collection (blog). Last updated March 13, 2013. http://curatethecollection.wordpress.com/

Flickr: The Commons. “About the Rights Statement.” Accessed June 1, 2014. https://www.flickr.com/commons/usage/

Frost, Olivia. "When the Object Is Digital: Properties of Digital Surrogate Objects and Implications for Learning.” In Museums in a Digital Age, edited by Ross Parry, 237-246. London: Routledge, 2010.

Google Cultural Institute. "Frequently Asked Questions.” Accessed June 15, 2014. http://www.google.com/culturalinstitute/about/

Ippolito, Jon. "Death by Wall Label." In New Media in the White Cube and Beyond, edited by Christiane Paul, 106-132. Berkeley: University of California Press, 2008.

Jeffries, Adrianne. "The Man behind Flickr on Making the Service 'Awesome Again."” The Verge. Last modified March 20, 2013. http://www.theverge.com/2013/3/20/4121574/flickr-chief-markus-spiering-talksphotos-and-marissa-mayer

Lowcountry Digital Library. “About the Lowcountry Digital Library.” Accessed June 20, 2014. http://lcdl.library.cofc.edu/about

Lowcountry Digital History Initiative. "The Charleston Hospital Workers Movement, 1968-1969.” Accessed June 20, 2014. 


\section{http://ldhi.library.cofc.edu/exhibits/show/charleston_hospital_workers_mo}

Manovich, Lev. The Language of New Media. Cambridge: MIT Press, 2001.

Manovich, Lev. introduction to "As We May Think” by Vannevar Bush, 35-36. In The

New Media Reader, edited by Noah Wardrip-Fruin and Nick Montfort.

Cambridge: MIT Press, 2003.

Manovich, Lev. Software Takes Command. New York: Bloomsbury, 2013.

McTavish, Lianne. "Visiting the Virtual Museum: Art and Experience Online." In New Museum Theory and Practice: An Introduction, edited by Janet Marstine, 226246. Oxford: Blackwell Publishing, 2006.

New Orleans Museum of Art. "Gordon Parks: The Making of an Argument.” Last updated August 27, 2013. http://noma.org/news/detail/222/GORDON-PARKSTHE-MAKING-OF-AN-ARGUMENT

Parry, Ross. Recoding the Museum: Digital Heritage and the Technologies of Change. London: Routledge, 2007.

Paul, Christiane. "Challenges for a Ubiquitous Museum: From the White Cube to the Black Box and Beyond." In New Media in the White Cube and Beyond, edited by Christiane Paul, 53-75. Berkeley: University of California Press, 2008.

Roussou, Maria. "Learning by Doing and Learning Through Play: An Exploration of Interactivity in Virtual Environments for Children." In Museums in a Digital Age, edited by Ross Parry, 247-265. London: Routledge, 2010.

Samis, Peter. “The Exploded Museum.” In Digital Technologies and the Museum Experience: Handheld Guides and Other Media, edited by Loic Tallon and Kevin Walker, 3-17. Lanham: AltaMira Press, 2008. 
Sassoon, Joanna. "Photographic Materiality in the Age of Digital Reproduction.” In Photographs Objects Histories: On the materiality of images, edited by Elizabeth Edwards and Janice Hart, 186-202. New York: Routledge, 2004.

Schleiner, Anne-Marie. "Fluidities and Oppositions among Curators, Filter Feeders and Future Artist." Intelligent Agent Magazine. January 2003. http://opensorcery.net/opposition.pdf

Sekula, Allan. "On the Invention of Photographic Meaning." In Thinking Photography, edited by Victor Burgin, 84-109. London: Macmillan Press, 1982.

Smith Bautista, Susana. Museums in the Digital Age: Changing Meanings of Place, Community, and Culture. Lanham: AltaMira Press, 2014. 